\title{
Decode-and-Forward Short-Packet Relaying in the Internet of Things: Timely Status Updates
}

\author{
Dongsheng Zheng, Yuli Yang, Senior Member, IEEE, Lai Wei, and Bingli Jiao, Senior Member, IEEE
}

\begin{abstract}
In this paper, a decode-and-forward (DF) shortpacket relaying model is developed to achieve timely status updates for intelligent monitoring within the Internet of Things (IoT), where the status updates generated at an IoT device are delivered to a remote server with the aid of a relay in both halfduplex (HD) and full-duplex (FD) modes. To characterise the data freshness of status updates, we exploit the age of information (AoI) as a metric, which is defined as the time elapsed since the generation of the latest successfully decoded status update. The average AoI is formulated and minimised for both HD-DF and FD-DF relaying IoT networks in finite blocklength regime. For the HD-DF relaying, we introduce a perfect approximation of the average AoI to solve the problem of average AoI minimisation with the optimal blocklengths in two phases. For the FD-DF relaying, we propose an iterative algorithm to solve the problem of average AoI minimisation by optimising the relay's transmit power and the blocklength. Illustrative numerical results not only substantiate the validity of our proposed algorithms, but also provide useful references for the IoT monitoring network design, specifically for the transmit power thresholds at the IoT device and the relay.
\end{abstract}

Index Terms-Age of information (AoI), decode-and-forward (DF), finite blocklength regime, full duplex (FD), half duplex (HD), short-packet relaying, status updates.

\section{INTRODUCTION}

$\mathbf{T}$ HE Internet of Things (IoT) is a promising paradigm to carry out ubiquitous connectivity of massive devices and implement a wide range of advanced applications in agriculture, industry, medicine, and allied sectors [1], [2]. As the monitoring infrastructure creates a foundation for IoT services, e.g., smart home, smart healthcare, intelligent transportation, industrial automation, etc., various types of sensors and actuators are deployed in IoT monitoring networks to collect and deliver the status updates of specific physical parameters such as temperature, humidity, wind strength, and so on [3], [4].

To provide accurate and effective services, an IoT monitoring network has to maintain the data freshness of status updates for the monitored physical parameters [5], [6]. The age of information (AoI) was proposed in the seminal works [7] and [8] as a new metric to measure the data freshness in a network. The way to improve the data freshness is to minimise

This work was supported in part by the National Key Research and Development Program of China under Grant 2020YFB1807802, Grant 2016ZX03001018-005, Grant 2019YFE0113200, and Grant 2018YFB2202202. (Corresponding author: Yuli Yang.)

D. Zheng, L. Wei and B. Jiao are with the Department of Electronics, Peking University, Beijing 100871, China (e-mail: zhengds@pku.edu.cn; future1997@pku.edu.cn; jiaobl@pku.edu.cn).

Y. Yang is with the School of Engineering, University of Lincoln, Lincoln LN6 7TS, U.K. (e-mail: yyang @lincoln.ac.uk). the AoI. Different from the metric to measure transmission delay, the concept of AoI characterises the timestamp of the latest successfully decoded status update at the destination. The state-of-the-art, the challenges and the future directions in the research of this fundamentally novel metric have been thoroughly surveyed in [9] and [10].

As a key measurement of transmission efficiency, the average AoI is used to evaluate the data freshness of status updates in a network from an ergodic perspective, which has been widely studied. The average AoI of G/G/1/1 systems was investigated in [11], with the service protocols concerning blocking and preemption. The average version age at each node in a network was evaluated in [12], where the status updates were delivered through a memoryless gossip protocol. The average AoI and average peak AoI of edge computing systems were analysed in [13], where sensor nodes firstly process the acquired information and then transmit the processed results to an edge receiver. The average AoI in energy harvesting wireless sensor networks was investigated in [14]-[16]. Under the constraints of average AoI and power consumption, the long-term average throughput was analysed and maximised in [17] given both perfect channel state information at the transmitter (CSIT) and statistical CSIT. Moreover, the impacts of quantization [18], source coding [19], channel coding [20], partial update [21], and selective encoding [22] on the AoI in various systems have been considered. Besides, a new performance metric for status updates, referred to as the age of incorrect information was developed in [23], to better capture the wrong information's deteriorating effect from the timelineand error-based perspective.

In practice, short-packet protocols have been exploited for the status updates in IoT networks to achieve ultra-reliable and low-latency communications [24], [25]. Recent informationtheoretic advances in the analysis in finite blocklength regime have established a basis for the design of short-packet communications [26]. Unlike the theoretical framework in infinite blocklength regime grounded upon Shannon's convergence of optimal coding rate to the error-free channel capacity, short-packet communications have to inevitably suffer from decoding error in the finite blocklength regime. Given a packet error probability, tight bounds on maximal coding rate of shortpacket communications have been derived in [27]. Based on these works, the AoI performance of short-packet communications have been studied in finite blocklength regime. For example, the average AoI of short-packet communications was investigated in [28] with various packet management schemes, including non-preemption scheme, preemption scheme, and retransmission scheme. In [29], the average AoI of status updates 
TABLE I

CONTRASTING THE NOVELTY OF OUR WORK TO THE LITERATURE.

\begin{tabular}{c|c|c|c|c}
\hline Contributions & This Work & [7]-[14], [16]-[22] & [28]-[30] & [15], [34], [35] \\
\hline \hline Age of Information (AoI) & $\checkmark$ & $\checkmark$ & $\checkmark$ & $\checkmark$ \\
\hline Finite Blocklength Regime & $\checkmark$ & & $\checkmark$ & \\
\hline Relay Networks & $\checkmark$ & & & $\checkmark$ \\
\hline Decode-and-Forward (DF) & $\checkmark$ & & & \\
\hline Half-Duplex (HD) Relaying & $\checkmark$ & & & $\checkmark$ \\
\hline Full-Duplex (FD) Relaying & $\checkmark$ & & & \\
\hline
\end{tabular}

was analysed and compared in finite blocklength regime for time-division and frequency-division multiple access systems. In [30], the average AoI of machine-type communications with short packets was developed and optimised, where the packet error probabilities were compared for the strategies of discarding and retransmitting the packets decoded unsuccessfully.

Furthermore, network densification is an influential approach to address the challenges of exponentially increasing data services and massive connectivity in the IoT [31]. The access point within a small cell takes on the role of a relay to forward the IoT devices' status updates to the remote server [32], [33]. Within macro cells, due to the transmit power limitations on IoT devices, their communications with the remote server also need to be implemented through the aid of a relay. Buyukates et al. investigated the average AoI of multihop multicast networks in [34], and Li et al. analysed the weighted average AoI of amplify-and-forward (AF) twoway relaying systems in [35].

As a common and dynamic topology in IoT wireless networks, the decode-and-forward (DF) relaying achieves better performance than the AF [36]-[38]. To the best of our knowledge, theoretical principles governing DF short-packet relaying IoT networks have not yet been addressed to assess their performance in maintaining the data freshness of status updates. Motivated by this, herein we study the AoI performance of DF short-packet relaying in finite blocklength regime, where the analytic expressions of average AoI are obtained for both half-duplex (HD) and full-duplex (FD) DF relaying IoT networks. Furthermore, we propose efficient algorithms to solve the optimisation problems for the average AoI minimisation through optimal designs of blocklength and transmit power.

The novelty of our work is compared with related studies of the AoI concept in Table I. In particular, our main contributions in this paper are three-fold:

- The HD-DF and FD-DF relaying models are developed to quantify the data freshness of status updates in the metric of AoI for the DF short-packet relaying IoT networks.

- The average AoI in finite blocklength regime is formulated for the HD-DF and FD-DF relaying IoT networks, with analytic expressions achieved.

- Efficient algorithms are proposed to solve the optimisation problems of the average AoI minimisation in HDDF and FD-DF relaying IoT networks, with the optimal designs of blocklength and transmit power.

To detail the above highlighted contributions, the remainder of this paper is organized as follows. In Section II, the system models of HD-DF and FD-DF relaying IoT networks are presented, followed by the introductions to the analysis in finite blocklength regime and the concept of AoI. Subsequently, Sections III and IV formulate and minimise the average AoI in finite blocklength regime for the HD-DF and FDDF relaying IoT networks, respectively. Illustrative numerical results are provided in Section V to substantiate our theoretical formulations and proposed algorithms for the average AoI minimisation. Finally, this paper is concluded in Section VI.

Notations: $f_{X}(x)$ and $F_{X}(x)$ stand for the probability density function (pdf) and the cumulative distribution function (cdf) of a random variable $X$, respectively. Moreover, $Q(x)=\int_{x}^{\infty}(1 / \sqrt{2 \pi}) \exp \left(-t^{2} / 2\right) d t$ is the Q-function, and $\mathbb{E}(\cdot)$ denotes the expectation (mean) operator. The greatest integer function and the least integer function are denoted by $\lfloor\cdot\rfloor$ and $\lceil\cdot\rceil$, respectively.

\section{System Model And PReliminaries}

In this section, the system models of HD-DF and FD-DF relaying networks are firstly presented. Then, the analysis of short-packet communications in finite blocklength regime and the definition of AoI are introduced.

\section{A. Decode-and-Forward Relaying}

Consider a DF relaying network shown in Fig. 1, where the status updates generated at an IoT device are delivered to a remote server with the aid of a relay. To achieve reliable transmissions of status updates over wireless channels, the automatic repeat request (ARQ) mechanism is adopted. Besides, both HD and FD modes are applied in the DF relaying.

1) HD-DF Relaying: In this mode, the transmission of each status update is composed of two phases, as shown in Fig. 1(a). In Phase 1, the IoT device transmits current status update to the relay. Once the relay successfully decodes the status update, it will send an acknowledgement (ACK) to the IoT device. If the IoT device does not receive an ACK before the predetermined timeout, it will retransmit this status update until receiving an ACK. In Phase 2, the relay transmits its decoded status update to the remote server whilst the IoT device keeps silent. Once the remote server successfully decodes the status update, an 


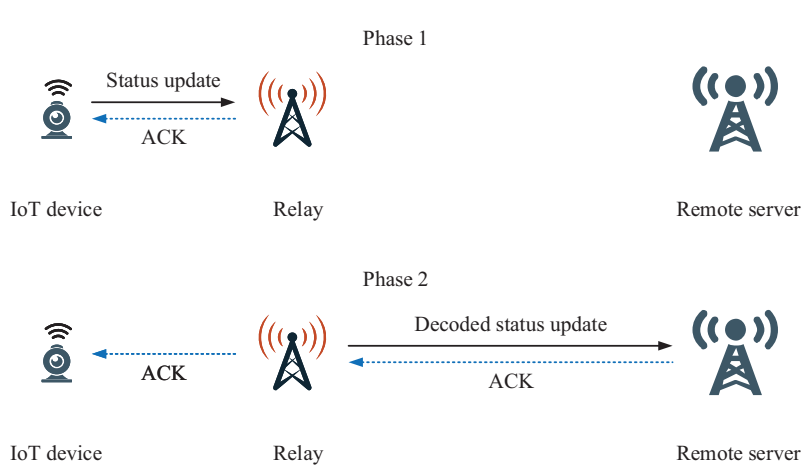

(a) Half-duplex relaying

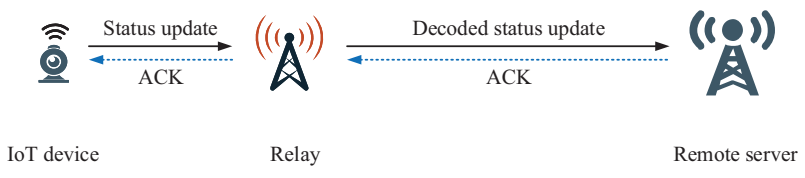

(b) Full-duplex relaying

Fig. 1. System model.

ACK is fed back to the relay and the IoT device, which triggers the IoT device to commence with the transmission of next status update. Otherwise, the relay will retransmit its currently decoded status update to the remote server until receiving an ACK.

2) FD-DF Relaying: In this mode, the relay receives the status update sent from the IoT device and, concurrently, transmits its decoded status update to the remote server, through the same time/frequency resource. In the link between the IoT device and the relay, an ACK fed back to the IoT device on the relay's successful decoding will trigger the IoT device's transmission of next status update. In the link between the relay and the remote server, the relay will be allowed to commence with the transmission of its next decoded status update once receiving an ACK from the remote server. However, the relay is likely to be ready for the transmission of its next decoded status update before receiving an ACK from the remote server. In this case, based on the preemption [28], the relay will replace its currently decoded status update with the succeeding one, even if the current one has not yet been successfully decoded by the remote server.

\section{B. Short-Packet Communications}

We investigate the DF relaying with short-packet communications in the finite blocklength regime, where the coding rate is denoted by $R=D / n$, with $D$ and $n$ standing for the number of information bits pertaining to a status update and the blocklength, respectively. Note that, $D$ is the same for both HD and FD modes, i.e., both modes use the same amount of information bits to characterise each status update.

A tight bound on the coding rate is given by [27]

$$
R=\log _{2}(1+\gamma)-\sqrt{\frac{V}{n}} \frac{Q^{-1}(\varepsilon)}{\ln 2},
$$

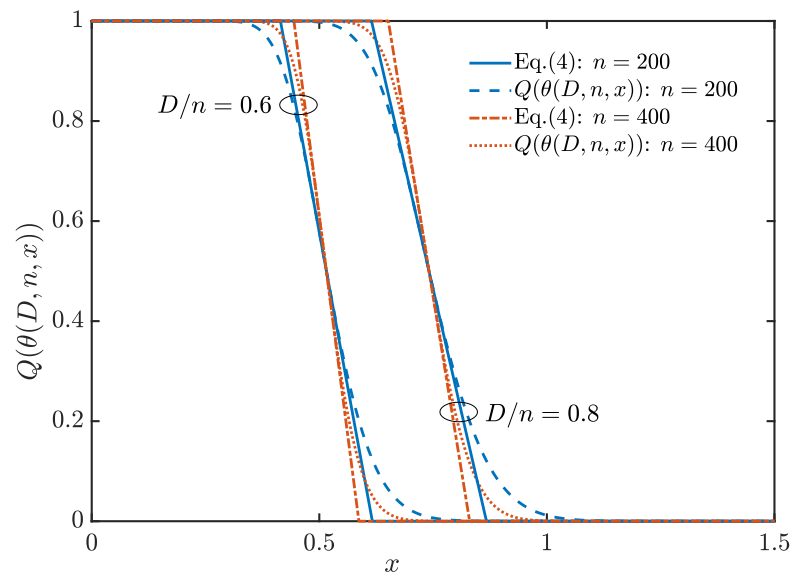

Fig. 2. The function $Q(\theta(D, n, x))$ in comparison with its approximation given by (4).

where $\gamma$ and $\varepsilon$ denote the signal-to-noise power ratio (SNR) and the packet error probability, respectively. Moreover, $V=$ $1-(1+\gamma)^{-2}$ is the channel dispersion, and $Q^{-1}(\cdot)$ is the inverse function of $Q(x)$.

From (1), the packet error probability $\varepsilon$ is formulated as

$$
\varepsilon=Q\left(\frac{(\ln 2) \sqrt{n}\left(\log _{2}(1+\gamma)-D / n\right)}{\sqrt{1-(1+\gamma)^{-2}}}\right) \triangleq Q(\theta(D, n, \gamma)),
$$

where the notation

$$
\theta(D, n, \gamma) \triangleq \frac{(\ln 2) \sqrt{n}\left(\log _{2}(1+\gamma)-D / n\right)}{\sqrt{1-(1+\gamma)^{-2}}}
$$

is used for the simplicity of expression.

In a general block-fading channel, the average packet error probability is calculated using

$$
\mathbb{E}(\varepsilon)=\int_{0}^{\infty} Q(\theta(D, n, x)) f_{\gamma}(x) \mathrm{d} x,
$$

where $f_{\gamma}(x)$ is the pdf of the SNR $\gamma$.

To gain the closed-form expression of $\mathbb{E}(\varepsilon)$, a linear approximation of Q-function is given by [39], [40]

$$
Q(\theta(D, n, x)) \approx \begin{cases}1, & x \leqslant \alpha-\frac{1}{2 \beta}, \\ \frac{1}{2}-\beta(x-\alpha), & \alpha-\frac{1}{2 \beta} \leqslant x \leqslant \alpha+\frac{1}{2 \beta}, \\ 0, & x \geqslant \alpha+\frac{1}{2 \beta},\end{cases}
$$

where $\alpha=2^{D / n}-1$ and $\beta=\sqrt{n /\left[2 \pi\left(2^{2 D / n}-1\right)\right]}$. To further validate the effectiveness of (4), we plot $Q(\theta(D, n, x))$ and its linear approximation in Fig. 2.

Substituting (4) into (3), we rewrite the average packet error probability as

$$
\mathbb{E}(\varepsilon)=\beta \int_{\alpha-1 /(2 \beta)}^{\alpha+1 /(2 \beta)} F_{\gamma}(x) \mathrm{d} x,
$$

where $F_{\gamma}(x)$ is the cdf of the SNR $\gamma$. 


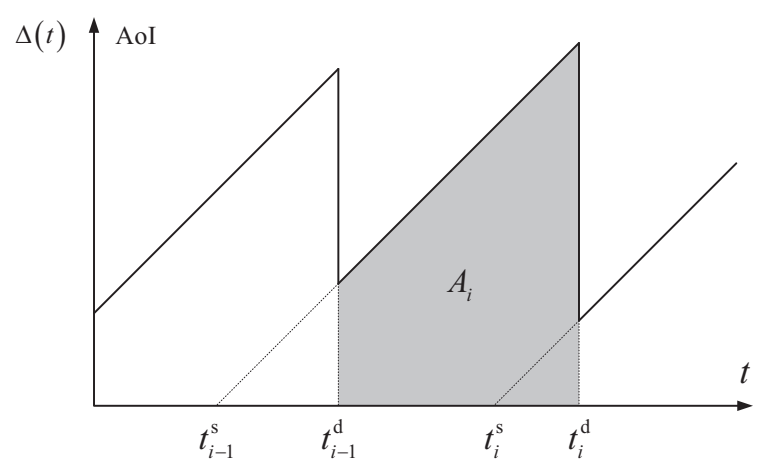

Fig. 3. An evolution of the AoI.

\section{Age of Information}

To measure the freshness of status updates in IoT networks, the metric AoI is defined as the time elapsed since the generation of the latest status update that has been successfully decoded at the remote server. Note that, the AoI increases linearly with time if there is no status update decoded successfully at the remote server. An evolution of the AoI under study is illustrated in Fig. 3, where $t_{i}^{\mathrm{s}}$ and $t_{i}^{\mathrm{d}}$ denote the generation time at the IoT device and the arrival time at the remote server, respectively, pertaining to the $i^{\text {th }}$ successful status update.

More specifically, the average AoI is expressed as

$$
\begin{aligned}
\bar{\Delta} & =\lim _{t \rightarrow \infty} \frac{1}{t} \int_{0}^{t} \Delta(t) \mathrm{d} t=\lim _{t \rightarrow \infty} \frac{1}{t} \sum_{i=1}^{N(t)} A_{i} \\
& =\lim _{t \rightarrow \infty} \frac{N(t)}{t} \mathbb{E}(A)=\lambda \mathbb{E}(A),
\end{aligned}
$$

where $\Delta(t)$ is the instantaneous AoI, and $N(t)$ is the number of status updates decoded successfully by the remote server at time $t$. Further, $\lambda=\lim _{t \rightarrow \infty} N(t) / t$ is defined as the rate of status updates decoded successfully at the remote server, and $A_{i}$, marked by the shadow in Fig. 3, is the right trapezoid area under the (waiting plus) delivery time of the $i^{\text {th }}$ status update. In addition, $\mathbb{E}(A)$ denotes the expectation of $A_{i}$, where the status update index $i$ is omitted since $A_{i}, i=1,2, \cdots$, are independent and identically distributed (i.i.d.) random variables from the ergodic perspective.

In this work, we will investigate the AoI in finite blocklength regime within HD-DF and FD-DF relaying networks based on the formulations of $\lambda$ and $\mathbb{E}(A)$.

\section{Rayleigh Fading Channels}

The majority of IoT infrastructures are deployed in rich scattering environments, for a wide range of applications in agriculture, industry, medicine, and allied sectors [1], [2]. In a non-line-of-sight scenario, such as a farm full of crops, a heavily built-up urban area, or a crowded corridor, Rayleigh fading is the most applicable model for the radio-frequency signal propagation [41], where the channel coefficient from a transmitter to a receiver is well-modelled as a random variable following a circularly-symmetric complex Gaussian distribu- tion and, hence, the magnitude of the channel coefficient is Rayleigh distributed [42].

Moreover, Rayleigh fading is the most popular model to facilitate the calculations in the analysis and optimisation of wireless systems and networks. Since the main purpose of this work is to investigate and compare the performance of HD-DF and FD-DF relaying IoT networks from the AoI perspective, the exploitation of Rayleigh fading model allows us to achieve analytic expressions for the evaluation and optimisation.

The cdf of a Rayleigh fading $\xi$, i.e., the magnitude of a channel coefficient, is given by

$$
F_{\xi}(\xi)=1-\exp \left(-\xi^{2} / H\right), \quad \xi \geqslant 0,
$$

where $H$ is the variance of the channel coefficient.

We remark that, the design principles and optimisation results obtained through the Rayleigh fading model in this work can be easily generalised into the scenarios modelled by Rician fading or Nakagami fading, if the signal propagation is dominated by a line-of-sight component in special applications or services.

\section{HALF-Duplex DeCODE-AND-ForWARD RELAYING}

In this section, the average AoI in the HD-DF relaying is formulated and minimised.

\section{A. Average AoI}

Within a HD-DF relaying IoT, the received SNRs at the relay and the remote server are given by

$$
\gamma_{\mathrm{r}}=h_{\mathrm{sr}} p_{\mathrm{s}} / \sigma^{2}
$$

and

$$
\gamma_{\mathrm{d}}=h_{\mathrm{rd}} p_{\mathrm{r}} / \sigma^{2},
$$

respectively, where $h_{\text {sr }}$ and $h_{\text {rd }}$ denote the channel gains from the IoT device to the relay and from the relay to the remote server, respectively. Moreover, $p_{\mathrm{s}}$ is the IoT device's transmit power, and $p_{\mathrm{r}}$ is the relay's transmit power. The variance of additive white Gaussian noise (AWGN) is $\sigma^{2}$.

In a Rayleigh-fading channel, the magnitude of channel coefficient, $\xi \in\left\{\sqrt{h_{\mathrm{sr}}}, \sqrt{h_{\mathrm{rd}}}\right\}$, obeys the Rayleigh distribution. Substituting $\xi^{2}=\gamma_{\mathrm{r}} /\left(p_{\mathrm{s}} / \sigma^{2}\right)$ and $\xi^{2}=\gamma_{\mathrm{d}} /\left(p_{\mathrm{r}} / \sigma^{2}\right)$ into (7), we have the cdfs of $\gamma_{\mathrm{r}}$ and $\gamma_{\mathrm{d}}$ expressed as

$$
F_{\gamma_{\mathrm{r}}}(x)= \begin{cases}1-\exp \left(-x / \bar{\gamma}_{\mathrm{r}}\right), & x \geqslant 0 \\ 0, & x<0\end{cases}
$$

and

$$
F_{\gamma_{\mathrm{d}}}(x)= \begin{cases}1-\exp \left(-x / \bar{\gamma}_{\mathrm{d}}\right), & x \geqslant 0, \\ 0, & x<0,\end{cases}
$$

respectively. Therefore, the received SNRs $\gamma_{\mathrm{r}}$ and $\gamma_{\mathrm{d}}$ follow the exponential distribution with means $\bar{\gamma}_{\mathrm{r}}=H_{\mathrm{sr}} p_{\mathrm{s}} / \sigma^{2}$ and $\bar{\gamma}_{\mathrm{d}}=H_{\mathrm{rd}} p_{\mathrm{r}} / \sigma^{2}$, where $H_{\mathrm{sr}}$ and $H_{\mathrm{rd}}$ are the variances of the channel coefficients.

Substituting (10) into (5), we obtain the relay's average packet error probability as

$\bar{\varepsilon}_{\mathrm{r}}=\beta_{\mathrm{r}} \int_{\alpha_{\mathrm{r}}-1 /\left(2 \beta_{\mathrm{r}}\right)}^{\alpha_{\mathrm{r}}+1 /\left(2 \beta_{\mathrm{r}}\right)}\left[1-\exp \left(-x / \bar{\gamma}_{\mathrm{r}}\right)\right] \mathrm{d} x$ 


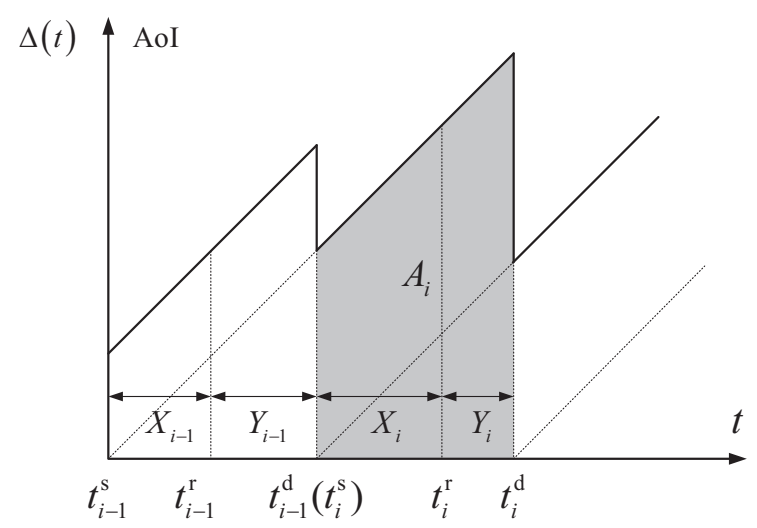

Fig. 4. An evolution of the AoI in HD-DF relaying networks.

$$
=1-\beta_{\mathrm{r}} \bar{\gamma}_{\mathrm{r}}\left[\exp \left(-\frac{\alpha_{\mathrm{r}}-1 /\left(2 \beta_{\mathrm{r}}\right)}{\bar{\gamma}_{\mathrm{r}}}\right)-\exp \left(-\frac{\alpha_{\mathrm{r}}+1 /\left(2 \beta_{\mathrm{r}}\right)}{\bar{\gamma}_{\mathrm{r}}}\right)\right],
$$

where $\alpha_{\mathrm{r}}=2^{D / n_{1}}-1$ and $\beta_{\mathrm{r}}=\sqrt{n_{1} /\left[2 \pi\left(2^{2 D / n_{1}}-1\right)\right]}$, with $n_{1}$ standing for the blocklength of each status update delivered in Phase 1.

Similarly, the remote server's average packet error probability is obtained by

$\bar{\varepsilon}_{\mathrm{d}}=1-\beta_{\mathrm{d}} \bar{\gamma}_{\mathrm{d}}\left[\exp \left(-\frac{\alpha_{\mathrm{d}}-1 /\left(2 \beta_{\mathrm{d}}\right)}{\bar{\gamma}_{\mathrm{d}}}\right)-\exp \left(-\frac{\alpha_{\mathrm{d}}+1 /\left(2 \beta_{\mathrm{d}}\right)}{\bar{\gamma}_{\mathrm{d}}}\right)\right]$, where $\alpha_{\mathrm{d}}=2^{D / n_{2}}-1$ and $\beta_{\mathrm{d}}=\sqrt{n_{2} /\left[2 \pi\left(2^{2 D / n_{2}}-1\right)\right]}$, with $n_{2}$ denoting the blocklength of each status update delivered in Phase 2.

For the $i^{\text {th }}$ status update decoded successfully at the relay, the number of total (re)transmissions in Phase 1 is denoted by $K_{\mathrm{r}, i}$. Obviously, $K_{\mathrm{r}, i}, i=1,2, \cdots$, are i.i.d. random variables having a Geometric distribution with the success probability $\left(1-\bar{\varepsilon}_{\mathrm{r}}\right)$. Thus, the mean and the second moment of $K_{\mathrm{r}, i}$ are

$$
\mathbb{E}\left(K_{\mathrm{r}}\right)=\frac{1}{1-\bar{\varepsilon}_{\mathrm{r}}}
$$

and

$$
\mathbb{E}\left(K_{\mathrm{r}}^{2}\right)=\frac{1+\bar{\varepsilon}_{\mathrm{r}}}{\left(1-\bar{\varepsilon}_{\mathrm{r}}\right)^{2}},
$$

respectively, where the index of status update, $i$, is omitted, without loss of generality.

Subsequently, the number of total (re)transmissions in Phase 2 for the $i^{\text {th }}$ status update decoded successfully at the remote server, is denoted by $K_{d, i}$, whose mean and second moment are

$$
\mathbb{E}\left(K_{\mathrm{d}}\right)=\frac{1}{1-\bar{\varepsilon}_{\mathrm{d}}}
$$

and

$$
\mathbb{E}\left(K_{\mathrm{d}}^{2}\right)=\frac{1+\bar{\varepsilon}_{\mathrm{d}}}{\left(1-\bar{\varepsilon}_{\mathrm{d}}\right)^{2}},
$$

respectively.

An evolution of the AoI in HD-DF relaying networks is illustrated in Fig. 4, where $t_{i}^{\mathrm{r}}$ denotes the arrival time of the $i^{\text {th }}$ status update decoded successfully at the relay. The shadow area pertaining to the $i^{\text {th }}$ status update, $A_{i}$, can be written as

$$
\begin{aligned}
A_{i} & =\left[\left(X_{i-1}+Y_{i-1}+X_{i}+Y_{i}\right)^{2}-\left(X_{i-1}+Y_{i-1}\right)^{2}\right] / 2 \\
& =\left(X_{i}^{2}+Y_{i}^{2}\right) / 2+X_{i} Y_{i}+\left(X_{i-1}+Y_{i-1}\right)\left(X_{i}+Y_{i}\right),
\end{aligned}
$$

where $X_{i}=K_{\mathrm{r}, i} T_{1}$ and $Y_{i}=K_{\mathrm{d}, i} T_{2}$ are the time durations for the delivery of the $i^{\text {th }}$ status update in Phase 1 and Phase 2, respectively, with $T_{1}$ and $T_{2}$ denoting the time durations of a single transmission in Phase 1 and Phase 2, respectively. Then, we have

$$
\begin{aligned}
\mathbb{E}(A)= & {\left[\mathbb{E}\left(K_{\mathrm{r}}^{2}\right)+2 \mathbb{E}^{2}\left(K_{\mathrm{r}}\right)\right] T_{1}^{2} / 2+\left[\mathbb{E}\left(K_{\mathrm{d}}^{2}\right)+2 \mathbb{E}^{2}\left(K_{\mathrm{d}}\right)\right] T_{2}^{2} / 2 } \\
& +3 \mathbb{E}\left(K_{\mathrm{r}}\right) T_{1} \mathbb{E}\left(K_{\mathrm{d}}\right) T_{2} \\
= & \frac{3}{2}\left(\frac{T_{1}}{1-\bar{\varepsilon}_{\mathrm{r}}}+\frac{T_{2}}{1-\bar{\varepsilon}_{\mathrm{d}}}\right)^{2}+\frac{\bar{\varepsilon}_{\mathrm{r}} T_{1}^{2}}{2\left(1-\bar{\varepsilon}_{\mathrm{r}}\right)^{2}}+\frac{\bar{\varepsilon}_{\mathrm{d}} T_{2}^{2}}{2\left(1-\bar{\varepsilon}_{\mathrm{d}}\right)^{2}} .
\end{aligned}
$$

Furthermore, the rate of status updates decoded successfully at the remote server, $\lambda$, is achieved at

$$
\lambda=\frac{1}{\mathbb{E}\left(K_{\mathrm{r}}\right) T_{1}+\mathbb{E}\left(K_{\mathrm{d}}\right) T_{2}}=\left(\frac{T_{1}}{1-\bar{\varepsilon}_{\mathrm{r}}}+\frac{T_{2}}{1-\bar{\varepsilon}_{\mathrm{d}}}\right)^{-1} .
$$

Substituting (19) and (20) into (6), we obtain the average AoI of HD-DF relaying networks as

$$
\begin{aligned}
\bar{\Delta}_{\mathrm{HD}} & =\frac{3}{2}\left(\frac{T_{1}}{1-\bar{\varepsilon}_{\mathrm{r}}}+\frac{T_{2}}{1-\bar{\varepsilon}_{\mathrm{d}}}\right)+\frac{\frac{\bar{\varepsilon}_{\mathrm{r}} T_{1}^{2}}{2\left(1-\bar{\varepsilon}_{\mathrm{r}}\right)^{2}}+\frac{\bar{\varepsilon}_{\mathrm{d}} T_{2}^{2}}{2\left(1-\bar{\varepsilon}_{\mathrm{d}}\right)^{2}}}{\frac{T_{1}}{1-\bar{\varepsilon}_{\mathrm{r}}}+\frac{T_{2}}{1-\bar{\varepsilon}_{\mathrm{d}}}} \\
& \approx \frac{3}{2}\left(\frac{T_{1}}{1-\bar{\varepsilon}_{\mathrm{r}}}+\frac{T_{2}}{1-\bar{\varepsilon}_{\mathrm{d}}}\right)+\frac{\frac{\bar{\varepsilon}_{\mathrm{r}} T_{1}^{2}}{2\left(1-\bar{\varepsilon}_{\mathrm{r}}\right)^{2}}}{\frac{2 T_{1}}{1-\bar{\varepsilon}_{\mathrm{r}}}}+\frac{\frac{\bar{\varepsilon}_{\mathrm{d}} T_{2}^{2}}{2\left(1-\bar{\varepsilon}_{\mathrm{d}}\right)^{2}}}{\frac{2 T_{2}}{1-\bar{\varepsilon}_{\mathrm{d}}}} \\
& =\frac{6+\bar{\varepsilon}_{\mathrm{r}}}{4\left(1-\bar{\varepsilon}_{\mathrm{r}}\right)} T_{1}+\frac{6+\bar{\varepsilon}_{\mathrm{d}}}{4\left(1-\bar{\varepsilon}_{\mathrm{d}}\right)} T_{2} \triangleq \tilde{\Delta}_{\mathrm{HD}},
\end{aligned}
$$

where the approximation (22) is obtained by assuming that the average transmission time of a packet in Phase 1 and that in Phase 2 are almost the same, i.e., $T_{1} /\left(1-\bar{\varepsilon}_{\mathrm{r}}\right) \approx T_{2} /\left(1-\bar{\varepsilon}_{\mathrm{d}}\right)$. It agrees with the reality of packet transmissions.

In Fig. 5, the expression given by (21) is compared with the Monte-Carlo simulation results of the average AoI in HD-DF relaying over $10^{6}$ status updates successfully decoded at the remote server, where $D=200$ information bits are conveyed through the blocklengths $n_{1}=n_{2}=200$ channel uses with the network bandwidth $B=1 \mathrm{MHz}$. The average channel gains $H_{\mathrm{sr}}=-85 \mathrm{~dB}$ and $H_{\mathrm{rd}}=-95 \mathrm{~dB}$. The AWGN variance is $\sigma^{2}=$ $-90 \mathrm{dBm}$. As shown in this figure, our derived average AoI expression (21) perfectly agrees with the simulation results. In the following, the average AoI of the HD-DF relaying will be minimised by optimising the blocklengths $n_{1}$ and $n_{2}$, on the basis of the expression (21).

\section{B. Average AoI Minimisation}

As shown in (12) and (13), higher received SNR results in lower packet error probability, thereby decreasing the average AoI. Hence, both the IoT device and the relay in the HD-DF relaying are encouraged to transmit signals at their maximum power, for the purpose of average AoI minimisation. 


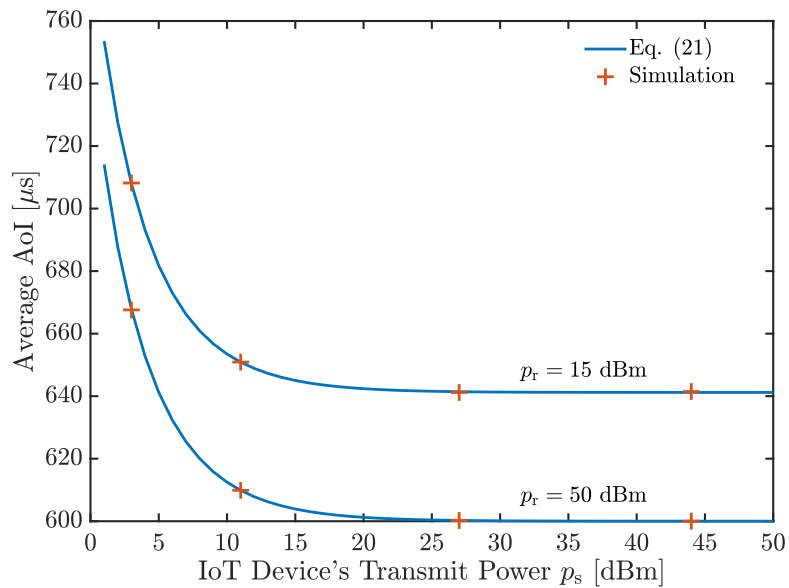

Fig. 5. The average AoI expression (21) in comparison with simulation results.

In the finite blocklength regime, larger blocklength leads to lower packet error probability but results in longer time duration for the transmission. Accordingly, given the transmit power at the IoT device and the relay, the average AoI of HDDF relaying networks is minimised through the optimisation of the blocklengths $n_{1}$ and $n_{2}$.

As it is tough to minimise the average AoI using the expression in (21), we will minimise its approximation given by (23). Further, the time durations of a single transmission can be expressed as $T_{1}=n_{1} / B$ and $T_{2}=n_{2} / B$, where $B$ is the bandwidth. Thus, the minimisation of average AoI is formulated by an optimisation problem as

$$
\begin{array}{ll}
\mathcal{P} 1: & \min _{n_{1}, n_{2}} \frac{6+\bar{\varepsilon}_{\mathrm{r}}}{4\left(1-\bar{\varepsilon}_{\mathrm{r}}\right)} \frac{n_{1}}{B}+\frac{6+\bar{\varepsilon}_{\mathrm{d}}}{4\left(1-\bar{\varepsilon}_{\mathrm{d}}\right)} \frac{n_{2}}{B} \\
\text { s.t. } & n_{1} \geqslant n_{\min }, \quad n_{1} \in \mathbb{Z}, \\
& n_{2} \geqslant n_{\min }, \quad n_{2} \in \mathbb{Z},
\end{array}
$$

where $n_{\min }$ is the predetermined minimum blocklength.

Referring to (12) and (13), we may find that the first item in (24) is only related to $n_{1}$ and that the second item in (24) is only related to $n_{2}$. Therefore, $\mathcal{P} 1$ can be decoupled into two independent optimisation subproblems whose objective functions are the first and second items in (24). We will solve them one by one.

Before stepping further into the optimisation of the first item in (24) subject to $n_{1}$, we present an approximation of the relay's average packet error probability, which has been validated in [30]. Based on (12), we have

$$
\begin{aligned}
\bar{\varepsilon}_{\mathrm{r}} & =1-\beta_{\mathrm{r}} \bar{\gamma}_{\mathrm{r}} \exp \left(-\frac{\alpha_{\mathrm{r}}+1 /\left(2 \beta_{\mathrm{r}}\right)}{\bar{\gamma}_{\mathrm{r}}}\right)\left[\exp \left(\frac{1}{\beta_{\mathrm{r}} \bar{\gamma}_{\mathrm{r}}}\right)-1\right] \\
& \approx 1-\exp \left(-\frac{\alpha_{\mathrm{r}}+1 /\left(2 \beta_{\mathrm{r}}\right)}{\bar{\gamma}_{\mathrm{r}}}\right) \\
& \approx 1-\exp \left(-\frac{2^{D / n_{1}}-1+\sqrt{\pi D \ln 2} / n_{1}}{\bar{\gamma}_{\mathrm{r}}}\right),
\end{aligned}
$$

where (25) and (26) are both achieved through the Taylor approximation of degree 1 . Note that, (25) holds for a moderate SNR $\bar{\gamma}_{\mathrm{r}}$, and (26) holds for a relatively small coding rate $D / n_{1}$.
The optimisation subproblem of the first item in (24) is formulated as

$$
\begin{array}{ll}
\mathcal{P} 2: & \min _{n_{1}} O_{1}\left(n_{1}\right) \\
\text { s.t. } & n_{1} \geqslant n_{\min }, \quad n_{1} \in \mathbb{Z},
\end{array}
$$

where the objective function $O_{1}\left(n_{1}\right)$ is given by

$$
O_{1}\left(n_{1}\right)=\frac{7 n_{1}}{4 B} \exp \left(\frac{2^{\frac{D}{n_{1}}}-1+\frac{\sqrt{\pi D \ln 2}}{n_{1}}}{\bar{\gamma}_{\mathrm{r}}}\right)-\frac{n_{1}}{4 B} .
$$

Relaxing the constraint $n_{1} \in \mathbb{Z}$ and allowing $n_{1}$ to take real values, i.e., $n_{1} \in \mathbb{R}$, we have the following lemma.

Lemma 1. The function $O_{1}\left(n_{1}\right)$ is convex with respect to $n_{1}$.

Proof: The first-order derivative and second-order derivative of $O_{1}\left(n_{1}\right)$ are given by

$$
O_{1}^{\prime}\left(n_{1}\right)=\frac{7}{4 B\left(1-\bar{\varepsilon}_{\mathrm{r}}\right)}\left(1-\frac{2^{\frac{D}{n_{1}}} D \ln 2+\sqrt{\pi D \ln 2}}{\bar{\gamma}_{\mathrm{r}} n_{1}}\right)-\frac{1}{4 B}
$$

and

$$
O_{1}^{\prime \prime}\left(n_{1}\right)=\frac{7(D \ln 2)^{2}}{4 B\left(1-\bar{\varepsilon}_{\mathrm{r}}\right) \bar{\gamma}_{\mathrm{r}} n_{1}^{3}}\left[2^{\frac{D}{n_{1}}}+\frac{\left(2^{\frac{D}{n_{1}}}+\sqrt{\frac{\pi}{D \ln 2}}\right)^{2}}{\bar{\gamma}_{\mathrm{r}}}\right]>0 .
$$

Therefore, the function $O_{1}\left(n_{1}\right)$ is convex with respect to $n_{1}$, which completes the proof.

According to Lemma 1, the optimal blocklength $n_{1}^{*}$ can be obtained by solving the Karush-Kuhn-Tucker (KKT) conditions. The Lagrangian is formulated as

$$
\mathcal{L}\left(O\left(n_{1}\right), \mu\right)=O_{1}\left(n_{1}\right)-\mu\left(n_{1}-n_{\min }\right),
$$

where $\mu$ is the Lagrange multiplier. Then, the KKT conditions are given by

$$
\begin{aligned}
\frac{\partial \mathcal{L}\left(O_{1}\left(n_{1}\right), \mu\right)}{\partial n_{1}}=O_{1}^{\prime}\left(n_{1}\right)-\mu & =0, \\
\mu\left(n_{1}-n_{\min }\right) & =0, \\
n_{1}-n_{\min } & \geqslant 0, \\
\mu & \geqslant 0 .
\end{aligned}
$$

Concerning the constraint $n_{1} \in \mathbb{Z}$, the optimal blocklength $n_{1}^{*}$ is obtained by

$$
n_{1}^{*}= \begin{cases}n_{\min }, & O_{1}^{\prime}\left(n_{\min }\right)>0, \\ \underset{n_{1} \in\left\{\left\lfloor n_{1}^{\star}\right\rfloor,\left[n_{1}^{\star}\right\rceil\right\}}{\arg \min } O_{1}\left(n_{1}\right), & O_{1}^{\prime}\left(n_{\min }\right) \leqslant 0,\end{cases}
$$

where $n_{1}^{\star}$ satisfies

$$
O_{1}^{\prime}\left(n_{1}^{\star}\right)=0,
$$

and can be obtained by the binary search algorithm.

Through the derivations similar with (26)-(36), we can obtain the optimal blocklength $n_{2}^{*}$ as

$$
n_{2}^{*}= \begin{cases}n_{\min }, & O_{2}^{\prime}\left(n_{\min }\right)>0, \\ \underset{n_{2} \in\left\{\left\lfloor n_{2}^{\star}\right\rfloor,\left\lceil n_{2}^{\star}\right\rceil\right\}}{\arg \min } O_{2}\left(n_{2}\right), & O_{2}^{\prime}\left(n_{\min }\right) \leqslant 0,\end{cases}
$$


where the function $O_{2}\left(n_{2}\right)$ and its first-order derivative $O_{2}^{\prime}\left(n_{2}\right)$ are given by

$$
O_{2}\left(n_{2}\right)=\frac{7 n_{2}}{4 B} \exp \left(\frac{2^{\frac{D}{n_{2}}}-1+\frac{\sqrt{\pi D \ln 2}}{n_{2}}}{\bar{\gamma}_{\mathrm{d}}}\right)-\frac{n_{2}}{4 B}
$$

and

$$
O_{2}^{\prime}\left(n_{2}\right)=\frac{7}{4 B\left(1-\bar{\varepsilon}_{\mathrm{d}}\right)}\left(1-\frac{2^{\frac{D}{n_{2}}} D \ln 2+\sqrt{\pi D \ln 2}}{\bar{\gamma}_{\mathrm{d}} n_{2}}\right)-\frac{1}{4 B},
$$

respectively. Moreover, $n_{2}^{\star}$ is the root of $O_{2}^{\prime}\left(n_{2}\right)=0$, which can be obtained by the binary search algorithm.

\section{Full-Duplex DeCODE-AND-Forward Relaying}

In this section, the average AoI in the FD-DF relaying is formulated and minimised.

\section{A. Average AoI}

In a FD-DF relaying IoT, the relay works in the FD mode and exploits self-interference (SI) cancellation techniques to mitigate the SI influence [43]-[45]. In this work, we consider the practical scenario with residual SI [46], [47]. Further, the blocklength in the link between the IoT device and the relay is assumed to be the same as that in the link between the relay and the remote server. Both are equal to $n \in \mathbb{Z}$.

When the relay receives the status update transmitted from the IoT device while forwarding its decoded status update to the remote server, its received signal, suffering from the SI, is expressed as

$$
y_{\mathrm{r}}^{\mathrm{SI}}=\sqrt{H_{\mathrm{sr}} p_{\mathrm{s}}} e_{\mathrm{sr}} x_{\mathrm{s}}+\sqrt{H_{\mathrm{rr}} p_{\mathrm{r}}} e_{\mathrm{rr}} x_{\mathrm{r}}+\omega_{1},
$$

where $H_{\text {sr }}$ denotes the average channel gain in the link between the IoT device and the relay, and $H_{\text {rr }}$ denotes the average channel gain of the relay's residual SI. Besides, $e_{\mathrm{sr}}$ and $e_{\mathrm{rr}}$ are both complex Gaussian variables with zero mean and unit variance. The signals transmitted from the IoT device and the relay are $x_{\mathrm{s}}$ and $x_{\mathrm{r}}$, respectively, and the AWGN is $\omega_{1} \sim \mathcal{C N}\left(0, \sigma^{2}\right)$.

In the case that the successful decoding at the remote server is completed earlier than that at the relay, the relay's received signal is free from the SI and obtained by

$$
y_{\mathrm{r}}^{\mathrm{nSI}}=\sqrt{H_{\mathrm{sr}} p_{\mathrm{s}}} e_{\mathrm{sr}} x_{\mathrm{s}}+\omega_{2},
$$

where $\omega_{2} \sim \mathcal{C N}\left(0, \sigma^{2}\right)$ is the AWGN.

The relay's received SNR in these two cases are given by

$$
\gamma_{\mathrm{r}}^{\mathrm{SI}}=\frac{H_{\mathrm{sr}} E_{\mathrm{sr}} p_{\mathrm{s}}}{H_{\mathrm{rr}} E_{\mathrm{rr}} p_{\mathrm{r}}+\sigma^{2}}
$$

and

$$
\gamma_{\mathrm{r}}^{\mathrm{nSI}}=\frac{H_{\mathrm{sr}} E_{\mathrm{sr}} p_{\mathrm{s}}}{\sigma^{2}},
$$

where $E_{\mathrm{sr}}$ and $E_{\mathrm{rr}}$ are both exponentially distributed random variables with unit mean.

The cdf of $\gamma_{\mathrm{r}}^{\mathrm{SI}}$ is obtained in [48] as

$$
F_{\gamma_{\mathrm{r}}^{\mathrm{SI}}}(x)= \begin{cases}1-\frac{1}{1+\left(\bar{\gamma}_{\mathrm{rr}} / \bar{\gamma}_{\mathrm{sr}}\right) x} \exp \left(-\frac{x}{\bar{\gamma}_{\mathrm{sr}}}\right), & x \geq 0, \\ 0, & x<0,\end{cases}
$$

where $\bar{\gamma}_{\mathrm{rr}}=H_{\mathrm{rr}} p_{\mathrm{r}} / \sigma^{2}$ and $\bar{\gamma}_{\mathrm{sr}}=H_{\mathrm{sr}} p_{\mathrm{s}} / \sigma^{2}$.

Substituting (45) into (5), we obtain the average packet error probability in the presence of residual SI as

$$
\begin{aligned}
\bar{\varepsilon}_{\mathrm{r}}^{\mathrm{SI}}= & \beta \int_{\alpha-1 /(2 \beta)}^{\alpha+1 /(2 \beta)}\left[1-\frac{1}{1+\left(\bar{\gamma}_{\mathrm{rr}} / \bar{\gamma}_{\mathrm{sr}}\right) x} \exp \left(-\frac{x}{\bar{\gamma}_{\mathrm{sr}}}\right)\right] \mathrm{d} x \\
= & 1-\frac{\beta \bar{\gamma}_{\mathrm{sr}}}{\bar{\gamma}_{\mathrm{rr}}} \exp \left(\frac{1}{\bar{\gamma}_{\mathrm{rr}}}\right) \mathrm{Ei}\left[-\frac{\alpha+1 /(2 \beta)}{\bar{\gamma}_{\mathrm{sr}}}-\frac{1}{\bar{\gamma}_{\mathrm{rr}}}\right] \\
& +\frac{\beta \bar{\gamma}_{\mathrm{sr}}}{\bar{\gamma}_{\mathrm{rr}}} \exp \left(\frac{1}{\bar{\gamma}_{\mathrm{rr}}}\right) \mathrm{Ei}\left[-\frac{\alpha-1 /(2 \beta)}{\bar{\gamma}_{\mathrm{sr}}}-\frac{1}{\bar{\gamma}_{\mathrm{rr}}}\right]
\end{aligned}
$$

where $\operatorname{Ei}(x)=\int_{-\infty}^{x}\left(e^{t} / t\right) \mathrm{d} t$ is the exponential integral.

Referring to (12), the average packet error probability in the case of no SI at the relay can be obtained by

$\bar{\varepsilon}_{\mathrm{r}}^{\mathrm{nSI}}=1-\beta \bar{\gamma}_{\mathrm{sr}}\left\{\exp \left[-\frac{\alpha-1 /(2 \beta)}{\bar{\gamma}_{\mathrm{sr}}}\right]-\exp \left[-\frac{\alpha+1 /(2 \beta)}{\bar{\gamma}_{\mathrm{sr}}}\right]\right\}$.

As there is no direct link between the IoT device and the remote server, the remote server's average packet error probability is achieved at

$$
\bar{\varepsilon}_{\mathrm{d}}=1-\beta \bar{\gamma}_{\mathrm{d}}\left\{\exp \left[-\frac{\alpha-1 /(2 \beta)}{\bar{\gamma}_{\mathrm{d}}}\right]-\exp \left[-\frac{\alpha+1 /(2 \beta)}{\bar{\gamma}_{\mathrm{d}}}\right]\right\},
$$

where $\bar{\gamma}_{\mathrm{d}}=H_{\mathrm{rd}} p_{\mathrm{r}} / \sigma^{2}$ with $H_{\mathrm{rd}}$ representing the average channel gain in the link between the relay and the remote server.

Before analysing the average AoI in the FD-DF relaying, we derive the mean and the second moment of the number of total (re)transmissions from the IoT device to the relay for a single status update decoded successfully at the relay, $K_{\mathrm{r}}$, which are given in Lemma 2.

Lemma 2. The mean and the second moment of $K_{\mathrm{r}}$ are formulated as

$$
\mathbb{E}\left(K_{\mathrm{r}}\right)=\frac{1+\bar{\varepsilon}_{\mathrm{r}}^{\mathrm{SI}}-\bar{\varepsilon}_{\mathrm{r}}^{\mathrm{nSI}}-\bar{\varepsilon}_{\mathrm{d}} \bar{\varepsilon}_{\mathrm{r}}^{\mathrm{SI}}}{\left(1-\bar{\varepsilon}_{\mathrm{r}}^{\mathrm{nSI}}\right)\left(1-\bar{\varepsilon}_{\mathrm{d}} \bar{\varepsilon}_{\mathrm{r}}^{\mathrm{SI}}\right)}
$$

and

$$
\begin{aligned}
\mathbb{E}\left(K_{\mathrm{r}}^{2}\right)= & \frac{\left(1+\bar{\varepsilon}_{\mathrm{r}}^{\mathrm{SI}}-\bar{\varepsilon}_{\mathrm{r}}^{\mathrm{nSI}}-\bar{\varepsilon}_{\mathrm{d}} \bar{\varepsilon}_{\mathrm{r}}^{\mathrm{SI}}\right)}{\left(1-\bar{\varepsilon}_{\mathrm{r}}^{\mathrm{nSI}}\right)\left(1-\bar{\varepsilon}_{\mathrm{d}} \bar{\varepsilon}_{\mathrm{r}}^{\mathrm{SI}}\right)}\left(\frac{2}{1-\bar{\varepsilon}_{\mathrm{r}}^{\mathrm{nSI}}}+\frac{2}{1-\bar{\varepsilon}_{\mathrm{d}} \bar{\varepsilon}_{\mathrm{r}}^{\mathrm{SI}}}-1\right) \\
& -\frac{2}{\left(1-\bar{\varepsilon}_{\mathrm{r}}^{\mathrm{nSI}}\right)\left(1-\bar{\varepsilon}_{\mathrm{d}} \bar{\varepsilon}_{\mathrm{r}}^{\mathrm{SI}}\right)} .
\end{aligned}
$$

respectively.

Proof: See Appendix A.

As shown in Fig. 6, the shadow area pertaining to the $i^{\text {th }}$ status update, $A_{i}$, is calculated using

$$
A_{i}=\left(X_{i-1}+M_{i}+Y_{i}\right)^{2} / 2-\left(X_{i-1}+Y_{i-1}\right)^{2} / 2,
$$

where $X_{i}=K_{\mathrm{r}, i} T_{\mathrm{FD}}$ and $Y_{i}=\mathbb{E}\left(K_{\mathrm{d}, i} \mid K_{\mathrm{d}, i} \leq K_{\mathrm{r}, i}\right) T_{\mathrm{FD}}$, with $T_{\mathrm{FD}}=n / B$ and $K_{\mathrm{d}, i}$ denoting the number of total (re)transmissions for the successful delivery of a single status update, without preemption, to the remote server. The random variable $M_{i}$ is the time gap between two consecutive status updates decoded successfully at the relay.

Lemma 3. The mean and the second moment of $M$ are 


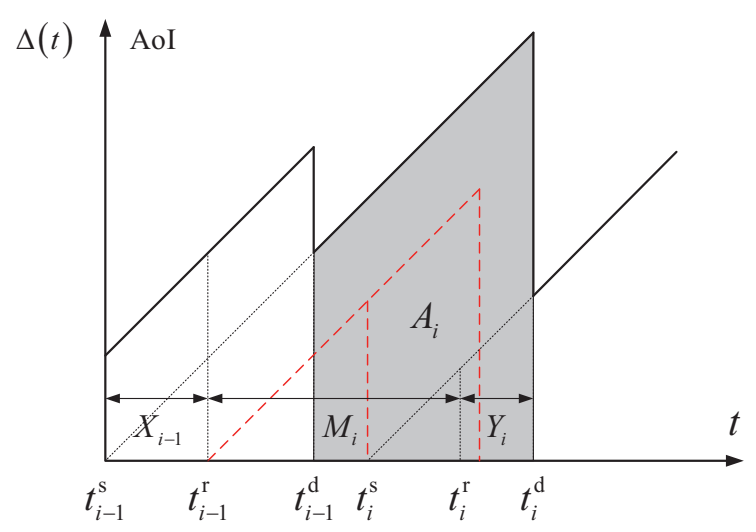

Fig. 6. An evolution of the AoI in FD-DF relaying networks, with preemption occurring in the relay's delivery of the $i^{\text {th }}$ status update.

formulated as

$$
\mathbb{E}(M)=\frac{\mathbb{E}\left(K_{\mathrm{r}}\right) T_{\mathrm{FD}}}{1-\eta}
$$

and

$$
\mathbb{E}\left(M^{2}\right)=\left[\frac{\mathbb{E}\left(K_{\mathrm{r}}^{2}\right)}{1-\eta}+\frac{2 \eta \mathbb{E}\left(K_{\mathrm{r}}\right) \mathbb{E}\left(K_{\mathrm{r}} \mid K_{\mathrm{r}}<K_{\mathrm{d}}\right)}{(1-\eta)^{2}}\right] T_{\mathrm{FD}}^{2},
$$

respectively, where $\eta$ denotes the probability that the relay's currently decoded status update is preempted by the succeeding one.

Proof: See Appendix B.

According to (51), the expectation of $A_{i}$ is calculated using $\mathbb{E}(A)=\mathbb{E}\left(M^{2}\right) / 2+\mathbb{E}(M) \mathbb{E}\left(K_{\mathrm{r}}\right) T_{\mathrm{FD}}+\mathbb{E}(M) \mathbb{E}\left(K_{\mathrm{d}} \mid K_{\mathrm{d}} \leq K_{\mathrm{r}}\right) T_{\mathrm{FD}}$.

Further, the rate of status updates decoded successfully at the remote server, $\lambda$, is written as $\lambda=1 / \mathbb{E}(M)$.

As a result, the average AoI in the FD-DF relaying is formulated as

$$
\begin{aligned}
& \bar{\Delta}_{\mathrm{FD}}=\lambda \mathbb{E}(A)=\frac{\mathbb{E}\left(M^{2}\right)}{2 \mathbb{E}(M)}+\mathbb{E}\left(K_{\mathrm{r}}\right) T_{\mathrm{FD}}+\mathbb{E}\left(K_{\mathrm{d}} \mid K_{\mathrm{d}} \leq K_{\mathrm{r}}\right) T_{\mathrm{FD}} \\
& =\left[\frac{\mathbb{E}\left(K_{\mathrm{r}}^{2}\right)}{2 \mathbb{E}\left(K_{\mathrm{r}}\right)}+\frac{\eta \mathbb{E}\left(K_{\mathrm{r}} \mid K_{\mathrm{r}}<K_{\mathrm{d}}\right)}{1-\eta}+\mathbb{E}\left(K_{\mathrm{r}}\right)+\mathbb{E}\left(K_{\mathrm{d}} \mid K_{\mathrm{d}} \leq K_{\mathrm{r}}\right)\right] T_{\mathrm{FD}}
\end{aligned}
$$

To obtain the analytic expression of $\bar{\Delta}_{\mathrm{FD}}$, we derive the probability $\eta$ and the conditional expectations $\mathbb{E}\left(K_{\mathrm{r}} \mid K_{\mathrm{r}}<K_{\mathrm{d}}\right)$, $\mathbb{E}\left(K_{\mathrm{d}} \mid K_{\mathrm{d}} \leq K_{\mathrm{r}}\right)$ as

$$
\begin{aligned}
& \eta=\operatorname{Pr}\left(K_{\mathrm{r}}<K_{\mathrm{d}}\right)=\sum_{k=2}^{\infty} \operatorname{Pr}\left(K_{\mathrm{d}}=k\right) \sum_{k^{\prime}=1}^{k-1} \operatorname{Pr}\left(K_{\mathrm{r}}=k^{\prime}\right) \\
&=\sum_{k=2}^{\infty}\left(\bar{\varepsilon}_{\mathrm{d}}\right)^{k-1}\left(1-\bar{\varepsilon}_{\mathrm{d}}\right) \sum_{k^{\prime}=1}^{k-1}\left(\bar{\varepsilon}_{\mathrm{r}}^{\mathrm{SI}}\right)^{k^{\prime}-1}\left(1-\bar{\varepsilon}_{\mathrm{r}}^{\mathrm{SI}}\right) \\
&=\frac{\bar{\varepsilon}_{\mathrm{d}}\left(1-\bar{\varepsilon}_{\mathrm{r}}^{\mathrm{SI}}\right)}{1-\bar{\varepsilon}_{\mathrm{d}} \bar{\varepsilon}_{\mathrm{r}}^{\mathrm{SI}}}, \\
& \mathbb{E}\left(K_{\mathrm{r}} \mid K_{\mathrm{r}}<K_{\mathrm{d}}\right)=\frac{\sum_{k=1}^{\infty} k \operatorname{Pr}\left(K_{\mathrm{r}}=k, K_{\mathrm{r}}<K_{\mathrm{d}}\right)}{\operatorname{Pr}\left(K_{\mathrm{r}}<K_{\mathrm{d}}\right)}
\end{aligned}
$$

$$
\begin{aligned}
& =\left(1-\bar{\varepsilon}_{\mathrm{d}} \bar{\varepsilon}_{\mathrm{r}}^{\mathrm{SI}}\right)^{-1}, \\
& \mathbb{E}\left(K_{\mathrm{d}} \mid K_{\mathrm{d}} \leq K_{\mathrm{r}}\right)=\frac{\sum_{k=1}^{\infty} k \operatorname{Pr}\left(K_{\mathrm{d}}=k, K_{\mathrm{d}} \leq K_{\mathrm{r}}\right)}{\operatorname{Pr}\left(K_{\mathrm{d}} \leq K_{\mathrm{r}}\right)} \\
& =\frac{1}{1-\eta} \sum_{k=1}^{\infty} k\left(\bar{\varepsilon}_{\mathrm{d}}\right)^{k-1}\left(1-\bar{\varepsilon}_{\mathrm{d}}\right)\left\{1-\sum_{k^{\prime}=1}^{k-1}\left(\bar{\varepsilon}_{\mathrm{r}}^{\mathrm{SI}}\right)^{k-1}\left(1-\bar{\varepsilon}_{\mathrm{r}}^{\mathrm{SI}}\right)\right\} \\
& =\left(1-\bar{\varepsilon}_{\mathrm{d}} \bar{\varepsilon}_{\mathrm{r}}^{\mathrm{SI}}\right)^{-1} .
\end{aligned}
$$

Substituting (49), (50), (55), (56), (57) into (54) and applying necessary arithmetic operations, we formulate the average AoI in the FD-DF relaying as

$$
\begin{aligned}
\bar{\Delta}_{\mathrm{FD}}= & \left(\frac{2}{1-\bar{\varepsilon}_{\mathrm{r}}^{\mathrm{nSI}}}+\frac{2}{1-\bar{\varepsilon}_{\mathrm{d}} \bar{\varepsilon}_{\mathrm{r}}^{\mathrm{SI}}}+\frac{1}{1-\bar{\varepsilon}_{\mathrm{d}}}-\frac{1}{2}\right) \frac{n}{B} \\
& -\left[\frac{1}{1+\bar{\varepsilon}_{\mathrm{r}}^{\mathrm{SI}}-\bar{\varepsilon}_{\mathrm{r}}^{\mathrm{nSI}}-\bar{\varepsilon}_{\mathrm{d}} \bar{\varepsilon}_{\mathrm{r}}^{\mathrm{SI}}}+\frac{1-\bar{\varepsilon}_{\mathrm{r}}^{\mathrm{SI}}}{\left(1-\bar{\varepsilon}_{\mathrm{r}}^{\mathrm{nSI}}\right)\left(1-\bar{\varepsilon}_{\mathrm{d}} \bar{\varepsilon}_{\mathrm{r}}^{\mathrm{SI}}\right)}\right] \frac{n}{B} \\
= & \frac{\bar{\varepsilon}_{\mathrm{r}}^{\mathrm{SI}}-\bar{\varepsilon}_{\mathrm{r}}^{\mathrm{nSI}}}{1-\bar{\varepsilon}_{\mathrm{d}} \bar{\varepsilon}_{\mathrm{r}}^{\mathrm{SI}}}\left(\frac{1}{1+\bar{\varepsilon}_{\mathrm{r}}^{\mathrm{SI}}-\bar{\varepsilon}_{\mathrm{r}}^{\mathrm{nSI}}-\bar{\varepsilon}_{\mathrm{d}} \bar{\varepsilon}_{\mathrm{r}}^{\mathrm{SI}}}+\frac{1}{1-\bar{\varepsilon}_{\mathrm{r}}^{\mathrm{nSI}}}\right) \frac{n}{B} \\
& +\left(\frac{2}{1-\bar{\varepsilon}_{\mathrm{r}}^{\mathrm{nSI}}}+\frac{1}{1-\bar{\varepsilon}_{\mathrm{d}}}-\frac{1}{2}\right) \frac{n}{B} \\
\approx & \frac{\bar{\varepsilon}_{\mathrm{r}}^{\mathrm{SI}}-\bar{\varepsilon}_{\mathrm{r}}^{\mathrm{nSI}}}{1-\bar{\varepsilon}_{\mathrm{d}}}\left(\frac{1}{1+\bar{\varepsilon}_{\mathrm{r}}^{\mathrm{SI}}}+\frac{1}{1-\bar{\varepsilon}_{\mathrm{r}}^{\mathrm{nSI}}}\right) \frac{n}{B} \\
& +\left(\frac{2}{1-\bar{\varepsilon}_{\mathrm{r}}^{\mathrm{nSI}}}+\frac{1}{1-\bar{\varepsilon}_{\mathrm{d}}}-\frac{1}{2}\right) \frac{n}{B} \triangleq \tilde{\Delta}_{\mathrm{FD}} .
\end{aligned}
$$

In Fig. 7, the expression (58) is plotted and compared with the Monte-Carlo simulation results of the average AoI in FDDF relaying over $10^{6}$ status updates successfully decoded at the remote server, where each status update contains $D=200$ information bits, conveyed through the blocklength $n=200$ channel uses with the network bandwidth $B=1 \mathrm{MHz}$. The average channel gains $H_{\mathrm{sr}}=-85 \mathrm{~dB}$ and $H_{\mathrm{rd}}=-95 \mathrm{~dB}$. The AWGN variance is $\sigma^{2}=-90 \mathrm{dBm}$. The average channel gain of the relay's residual SI $H_{r r}=-115 \mathrm{~dB}$. As shown in this figure, our derived average AoI expression perfectly agrees with the simulation results. In the following, the average AoI of the FD-DF relaying will be minimised by optimising the relay's transmit power $p_{\mathrm{r}}$ and the blocklength $n$, on the basis of the expression (58).

\section{B. Average AoI Minimisation}

To minimise the average AoI in the FD-DF relaying, the IoT device is encouraged to transmit signals at its maximum power, while the relay's transmit power needs to be optimised. Apparently, higher transmit power at the relay leads to lower packet error probability at the remote server and, however, higher packet error probability at itself due to the SI.

The complicated expression of average AoI $\bar{\Delta}_{\mathrm{FD}}$ in (58) impedes further optimisation of the relay's transmit power and the blocklength. Thus, we will minimise the average AoI based on the approximation $\tilde{\Delta}_{\mathrm{FD}}$ in (59). Given the transmit power at 


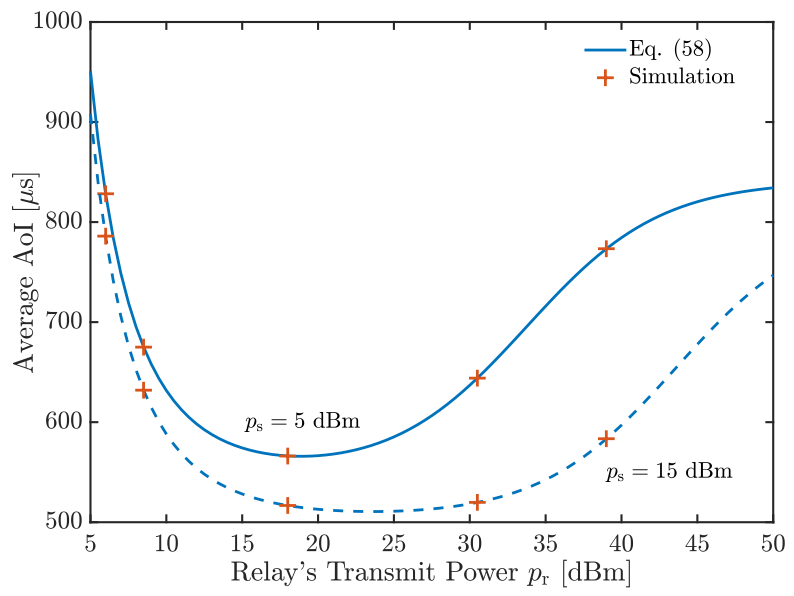

Fig. 7. The average AoI expression (58) in comparison with simulation results.

the IoT device, the minimisation of average AoI in the FD-DF relaying is formulated by an optimisation problem as

$$
\begin{array}{ll}
\text { P3: } & \min _{n, p_{\mathrm{r}}} \tilde{\Delta}_{\mathrm{FD}} \\
\text { s.t. } & n \geqslant n_{\min }, \quad n \in \mathbb{Z} \\
& 0<p_{\mathrm{r}} \leqslant p_{\mathrm{r}}^{\max },
\end{array}
$$

where $p_{\mathrm{r}}^{\max }$ is the relay's maximum transmit power.

Referring to the approximation (26), $\bar{\varepsilon}_{\mathrm{r}}^{\mathrm{nSI}}$ in (47) and $\bar{\varepsilon}_{\mathrm{d}}$ in (48) are approximated to

$$
\bar{\varepsilon}_{\mathrm{r}}^{\mathrm{nSI}} \approx 1-\exp \left[-\frac{b(n)}{\bar{\gamma}_{\mathrm{sr}}}\right]
$$

and

$$
\bar{\varepsilon}_{\mathrm{d}} \approx 1-\exp \left[-\frac{b(n)}{\bar{\gamma}_{\mathrm{d}}}\right]
$$

through the Taylor approximation of degree 1 as well, where $b(n)=2^{D / n}-1+\sqrt{\pi D \ln 2} / n$ is used for the simplicity of expression.

Moreover, $\bar{\varepsilon}_{\mathrm{r}}^{\mathrm{SI}}$ is approximated as

$$
\begin{aligned}
& \bar{\varepsilon}_{\mathrm{r}}^{\mathrm{SI}}=1-\beta \int_{\alpha-1 /(2 \beta)}^{\alpha+1 /(2 \beta)} \frac{1}{1+\left(\bar{\gamma}_{\mathrm{rr}} / \bar{\gamma}_{\mathrm{sr}}\right) x} \exp \left(-\frac{x}{\bar{\gamma}_{\mathrm{sr}}}\right) \mathrm{d} x \\
& \approx 1-\frac{1}{1+\left(\bar{\gamma}_{\mathrm{rr}} / \bar{\gamma}_{\mathrm{sr}}\right)[\alpha+1 /(2 \beta)]} \exp \left(-\frac{\alpha+1 /(2 \beta)}{\bar{\gamma}_{\mathrm{sr}}}\right) \\
& \approx 1-\frac{\bar{\gamma}_{\mathrm{sr}}}{\bar{\gamma}_{\mathrm{sr}}+\bar{\gamma}_{\mathrm{rr}} b(n)} \exp \left(-\frac{b(n)}{\bar{\gamma}_{\mathrm{sr}}}\right)
\end{aligned}
$$

where the approximation (63) is obtained by replacing the integrand with $e^{-[\alpha+1 /(2 \beta)] / \bar{\gamma}_{\mathrm{sr}}} /\left(1+\left(\bar{\gamma}_{\mathrm{rr}} / \bar{\gamma}_{\mathrm{sr}}\right)[\alpha+1 /(2 \beta)]\right)$ due to the small interval length $1 / \beta$ of the integration, and (64) is achieved through the Taylor approximation of degree 1.

Next, we adopt a low-complexity iterative algorithm to solve $\mathcal{P} 3$, i.e., two optimisation subproblems are characterised for the relay's optimal transmit power given the blocklength and the optimal blocklength given the relay's transmit power.

1) Optimal Transmit Power at the Relay: Given the blocklength $n$, the average packet error probabilities $\bar{\varepsilon}_{\mathrm{r}}^{\mathrm{SI}}, \bar{\varepsilon}_{\mathrm{r}}^{\mathrm{nSI}}$ and $\bar{\varepsilon}_{\mathrm{d}}$ are simplified as

$$
\begin{gathered}
\bar{\varepsilon}_{\mathrm{r}}^{\mathrm{SI}}=1-\frac{a}{1+k \bar{\gamma}_{\mathrm{d}}}, \\
\bar{\varepsilon}_{\mathrm{r}}^{\mathrm{nSI}}=1-a, \\
\bar{\varepsilon}_{\mathrm{d}}=1-\exp \left(-\frac{b}{\bar{\gamma}_{\mathrm{d}}}\right),
\end{gathered}
$$

where $a=\exp \left[-\left(2^{D / n}-1+\sqrt{\pi D \ln 2} / n\right) / \bar{\gamma}_{\mathrm{sr}}\right], b=2^{D / n}-$ $1+\sqrt{\pi D \ln 2} / n$ and $k=b H_{\mathrm{rr}} /\left(H_{\mathrm{rd}} \bar{\gamma}_{\mathrm{sr}}\right)$ are all constants. As the remote server's average SNR $\bar{\gamma}_{\mathrm{d}}=H_{\mathrm{rd}} p_{\mathrm{r}} / \sigma^{2}$, the subproblem of the relay's optimal transmit power is expressed as

$$
\begin{aligned}
& \mathcal{P} 4: \min _{\bar{\gamma}_{\mathrm{d}}} O_{3}\left(\bar{\gamma}_{\mathrm{d}}\right) \\
& \text { s.t. } \quad(60 \mathrm{~b}),
\end{aligned}
$$

where the objective function $O_{3}\left(\bar{\gamma}_{\mathrm{d}}\right)$ is

$$
\begin{aligned}
O_{3}\left(\bar{\gamma}_{\mathrm{d}}\right) & =\frac{\bar{\varepsilon}_{\mathrm{r}}^{\mathrm{SI}}-\bar{\varepsilon}_{\mathrm{r}}^{\mathrm{nSI}}}{1-\bar{\varepsilon}_{\mathrm{d}}}\left(\frac{1}{1+\bar{\varepsilon}_{\mathrm{r}}^{\mathrm{SI}}}+\frac{1}{1-\bar{\varepsilon}_{\mathrm{r}}^{\mathrm{nSI}}}\right)+\frac{1}{1-\bar{\varepsilon}_{\mathrm{d}}} \\
& =\left[3-\frac{(2-a)\left(1+k \bar{\gamma}_{\mathrm{d}}\right)}{2+2 k \bar{\gamma}_{\mathrm{d}}-a}-\frac{1}{1+k \bar{\gamma}_{\mathrm{d}}}\right] \exp \left(\frac{b}{\bar{\gamma}_{\mathrm{d}}}\right) .
\end{aligned}
$$

The first-order derivative of $O_{3}\left(\bar{\gamma}_{\mathrm{d}}\right)$ is given by

$$
O_{3}^{\prime}\left(\bar{\gamma}_{\mathrm{d}}\right)=G\left(\bar{\gamma}_{\mathrm{d}}\right) \frac{\exp \left(b / \bar{\gamma}_{\mathrm{d}}\right)}{\bar{\gamma}_{\mathrm{d}}^{2}\left(1+k \bar{\gamma}_{\mathrm{d}}\right)^{2}\left(2+2 k \bar{\gamma}_{\mathrm{d}}-a\right)^{2}},
$$

where $G\left(\bar{\gamma}_{\mathrm{d}}\right)$ is defined as

$$
G\left(\bar{\gamma}_{\mathrm{d}}\right)=\kappa_{1} \bar{\gamma}_{\mathrm{d}}^{4}+\kappa_{2} \bar{\gamma}_{\mathrm{d}}^{3}+\kappa_{3} \bar{\gamma}_{\mathrm{d}}^{2}+\kappa_{4} \bar{\gamma}_{\mathrm{d}}+\kappa_{5}
$$

with

$$
\begin{aligned}
& \kappa_{1}=\left(4+2 a-8 b k-2 a b k-a^{2}\right) k^{3}, \\
& \kappa_{2}=\left(8+2 a b k+a^{2} b k-28 b k-2 a^{2}\right) k^{2}, \\
& \kappa_{3}=(4+14 a b k-2 a-36 b k) k, \\
& \kappa_{4}=\left(14 a b-20 b-2 a^{2} b\right) k, \\
& \kappa_{5}=-b(2-a)^{2} .
\end{aligned}
$$

As $\bar{\gamma}_{\mathrm{d}}^{-2}\left(1+k \bar{\gamma}_{\mathrm{d}}\right)^{-2}\left(2+2 k \bar{\gamma}_{\mathrm{d}}-a\right)^{-2} \exp \left(b / \bar{\gamma}_{\mathrm{d}}\right)>0$, the monotonicity of the objective function $O_{3}\left(\bar{\gamma}_{\mathrm{d}}\right)$ can be derived from $G\left(\bar{\gamma}_{\mathrm{d}}\right)$. Moreover, we have $G(0)=-b(2-a)^{2}<0$. Let $\mathcal{R}$ be the set containing all positive real roots of the equation $G\left(\bar{\gamma}_{\mathrm{d}}\right)=0$, which can be obtained by the general formula for solving quartic equations. Consequently, the solution to $\mathcal{P} 4$ is

$$
p_{\mathrm{r}}^{*}= \begin{cases}p_{\mathrm{r}}^{\max }, & H_{\mathrm{rd}} p_{\mathrm{r}}^{\max } / \sigma^{2}<\bar{\gamma}_{\mathrm{d}}^{\min }, \\ p_{\mathrm{r}}^{\star}, & \text { otherwise }\end{cases}
$$

where $\bar{\gamma}_{\mathrm{d}}^{\min }$ is the smallest element in the set $\mathcal{R}$ if $\mathcal{R} \neq \varnothing$, and $\bar{\gamma}_{\mathrm{d}}^{\min } \triangleq+\infty$ if $\mathcal{R}=\emptyset$. Meanwhile, $p_{\mathrm{r}}^{\star}$ is obtained by

$$
p_{\mathrm{r}}^{\star}=\underset{\bar{\gamma}_{\mathrm{d}} \in \mathcal{R}, \bar{\gamma}_{\mathrm{d}} \leq H_{\mathrm{rd}} \max _{\mathrm{r}}^{\max } / \sigma^{2}}{\arg \min } G\left(\bar{\gamma}_{\mathrm{d}}\right) .
$$

2) Optimal Blocklength: Given the relay's transmit power $p_{\mathrm{r}}$, the average channel gains $\bar{\gamma}_{\mathrm{d}}$ and $\bar{\gamma}_{\mathrm{rr}}$ are fixed. As the average SNRs $\bar{\gamma}_{\mathrm{sr}}, \bar{\gamma}_{\mathrm{rr}}, \bar{\gamma}_{\mathrm{d}}$ are all constants, the subproblem of the optimal blocklength is formulated as

$$
\text { P5 : } \min _{n} O_{4}(n)
$$




$$
\text { s.t. }(60 a) \text {, }
$$

where the objective function $O_{4}(n)$ can be written as

$$
\begin{aligned}
O_{4}(n)= & \frac{\bar{\varepsilon}_{\mathrm{r}}^{\mathrm{SI}}-\bar{\varepsilon}_{\mathrm{r}}^{\mathrm{nSI}}}{1-\bar{\varepsilon}_{\mathrm{d}}}\left(\frac{1}{1+\bar{\varepsilon}_{\mathrm{r}}^{\mathrm{SI}}}+\frac{1}{1-\bar{\varepsilon}_{\mathrm{r}}^{\mathrm{nSI}}}\right) n \\
& +\left(\frac{2}{1-\bar{\varepsilon}_{\mathrm{r}}^{\mathrm{nSI}}}+\frac{1}{1-\bar{\varepsilon}_{\mathrm{d}}}-\frac{1}{2}\right) n \\
= & {\left[\frac{\rho b \exp \left(b / \bar{\gamma}_{\mathrm{d}}\right)}{2(1+\rho b) \exp \left(b / \bar{\gamma}_{\mathrm{sr}}\right)-1}+\frac{\rho b \exp \left(b / \bar{\gamma}_{\mathrm{d}}\right)}{1+\rho b}\right] n } \\
& +\left[2 \exp \left(\frac{b}{\bar{\gamma}_{\mathrm{sr}}}\right)+\exp \left(\frac{b}{\bar{\gamma}_{\mathrm{d}}}\right)-\frac{1}{2}\right] n
\end{aligned}
$$

with $\rho=\bar{\gamma}_{\mathrm{rr}} / \bar{\gamma}_{\mathrm{sr}}$ is used for the simplicity of expression.

Note that, the objective function $O_{4}(n)$ is not necessarily convex with respect to the blocklength $n$. To solve this subproblem effectively, we first derive an upper bound on $n$. If the blocklength exceeds this upper bound, $O_{4}(n)$ will increase monotonically. As such, the range of optimal blocklength is shortened. Then, we utilise an exhaustive algorithm to obtain the optimal blocklength $n^{*}$.

To begin with, the first-order derivatives of $\bar{\varepsilon}_{\mathrm{d}}, \bar{\varepsilon}_{\mathrm{r}}^{\mathrm{nSI}}$ and $\bar{\varepsilon}_{\mathrm{r}}^{\mathrm{SI}}$ are given by

$$
\begin{gathered}
\bar{\varepsilon}_{\mathrm{d}}^{\prime}=\frac{b^{\prime}}{\bar{\gamma}_{\mathrm{d}}}\left(1-\bar{\varepsilon}_{\mathrm{d}}\right), \\
\left(\bar{\varepsilon}_{\mathrm{r}}^{\mathrm{nSI}}\right)^{\prime}=\frac{b^{\prime}}{\bar{\gamma}_{\mathrm{sr}}}\left(1-\bar{\varepsilon}_{\mathrm{r}}^{\mathrm{nSI}}\right), \\
\left(\bar{\varepsilon}_{\mathrm{r}}^{\mathrm{SI}}\right)^{\prime}=\frac{\left(1+\rho b+\rho \bar{\gamma}_{\mathrm{sr}}\right) b^{\prime}}{(1+\rho b) \bar{\gamma}_{\mathrm{sr}}}\left(1-\bar{\varepsilon}_{\mathrm{r}}^{\mathrm{SI}}\right),
\end{gathered}
$$

where $b^{\prime}(n)=-(\ln 2) 2^{D / n} D / n^{2}-\sqrt{\pi D \ln 2} / n^{2}<0$. Then, the first-order derivative of $\mathrm{O}_{4}(n)$ is given in (78), at the top of next page.

As $n b^{\prime}(n)=-(\ln 2) 2^{D / n} D / n-\sqrt{\pi D \ln 2} / n$ is a monotonically increasing function of $n$, the objective function $O_{4}(n)$ increases monotonically when the blocklength $n$ satisfies

$$
\left\{\begin{array}{l}
3+4 n b^{\prime}(n) / \bar{\gamma}_{\mathrm{sr}}>0, \\
1+\left(4 / \bar{\gamma}_{\mathrm{d}}+2 / \bar{\gamma}_{\mathrm{sr}}+2 \rho\right) n b^{\prime}(n)>0, \\
n \geq n_{\min }, \quad n \in \mathbb{Z}
\end{array}\right.
$$

The minimum value of $n$ satisfying inequalities (79) is denoted by $n^{\star}$ and can be obtained through the binary search algorithm, which shortens the range of the optimal blocklength to $\left[n_{\min }, n^{\star}\right]$. Subsequently, an exhaustive search algorithm is ready to find the optimal blocklength $n^{*}$. Our proposed iterative algorithm conceived for solving the minimisation problem $\mathcal{P} 3$ is summarised in Algorithm 1.

\section{NumericAl RESUltS}

In this section, illustrative numerical results are provided to compare the AoI performance of HD-DF and FD-DF relaying IoT networks, where the default parameters are set as follows. The network bandwidth is $B=1 \mathrm{MHz}$. For a status update, there are $D=200$ information bits conveyed by the minimum blocklength $n_{\min }=100$ channel uses. The AWGN variance is $\sigma^{2}=-90 \mathrm{dBm}$. The average channel gain between the IoT device and the relay is $H_{\mathrm{sr}}=-85 \mathrm{~dB}$, whilst the average

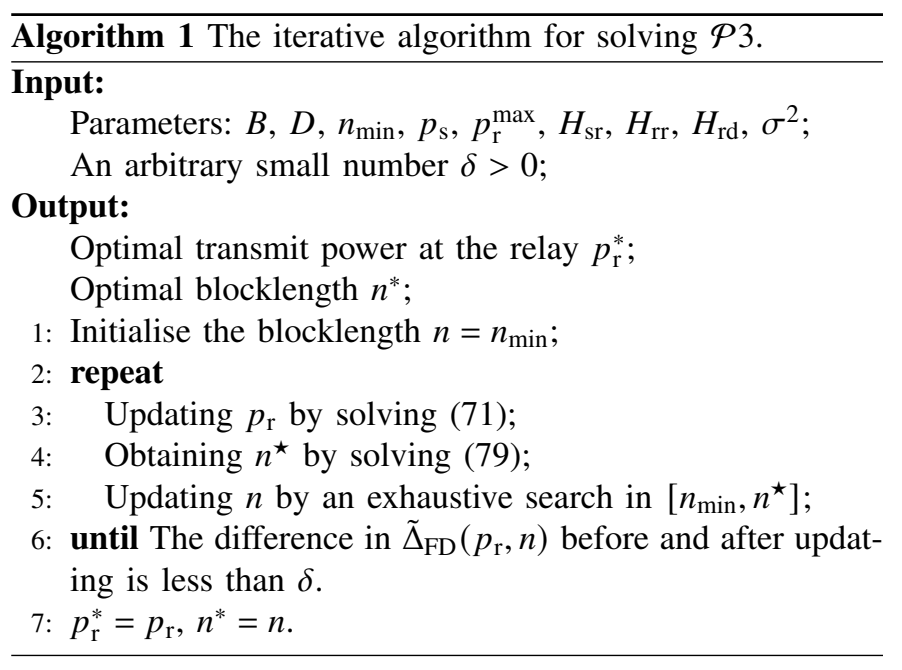

channel gain between the relay and the remote server is $H_{\text {rd }}=-95 \mathrm{~dB}$. In the HD-DF relaying IoT networks, the relay transmits its successfully decoded status updates at the maximum power $p_{\mathrm{r}}^{\max }$ to minimise the average AoI.

To begin with, the average AoI in HD-DF relaying, $\bar{\Delta}_{\mathrm{HD}}$ in (21), versus the blocklengths $n_{1}$ and $n_{2}$ in Phases 1 and 2, is plotted in Fig. 8, where the IoT device's transmit power $p_{\mathrm{s}}=5 \mathrm{dBm}$ and the relay's transmit power $p_{\mathrm{r}}=15 \mathrm{dBm}$. The average AoI in FD-DF relaying, $\bar{\Delta}_{\text {FD }}$ in (58), versus the blocklength $n$ and the relay's transmit power $p_{\mathrm{r}}$, is plotted in Fig. 9, where $p_{\mathrm{s}}=5 \mathrm{dBm}$ and the average channel gain of the relay's residual SI $H_{\mathrm{rr}}=-115 \mathrm{~dB}$.

To illustrate the validity of the approximation $\bar{\Delta}_{\mathrm{HD}} \approx \tilde{\Delta}_{\mathrm{HD}}$ in (23) and ensuing optimisation of the blocklengths, we plot the minimised average AoI of HD-DF relaying, versus the IoT device's transmit power $p_{\mathrm{s}}$, in Fig. 10, where the optimal blocklengths $n_{1}^{*}$ and $n_{2}^{*}$ are obtained by (36) and (38), respectively. For the sake of comparison, the optimal blocklengths $n_{1}^{\dagger}$ and $n_{2}^{\dagger}$ are obtained through exhaustive search upon (21). As shown in this figure, the minimised results of the expression $\bar{\Delta}_{\mathrm{HD}}\left(n_{1}^{*}, n_{2}^{*}\right)$ in (21) are matched perfectly by those of its approximation $\tilde{\Delta}_{\mathrm{HD}}\left(n_{1}^{*}, n_{2}^{*}\right)$ in (23). Moreover, the minimised average AoI results obtained by our proposed optimisation, $\bar{\Delta}_{\mathrm{HD}}\left(n_{1}^{*}, n_{2}^{*}\right)$ and $\tilde{\Delta}_{\mathrm{HD}}\left(n_{1}^{*}, n_{2}^{*}\right)$, perfectly agree with the optimal solution via exhaustive search $\bar{\Delta}_{\mathrm{HD}}\left(n_{1}^{\dagger}, n_{2}^{\dagger}\right)$, which substantiates the credibility of our optimised blocklengths $n_{1}^{*}$ and $n_{2}^{*}$.

For the FD-DF relaying IoT networks, to validate the effectiveness of the approximation $\tilde{\Delta}_{\mathrm{FD}}\left(p_{\mathrm{r}}, n\right)$ in (59) and the iterative algorithm given in Algorithm 1, we compare the minimised average AoI results obtained by our iterative algorithm, including $\bar{\Delta}_{\mathrm{FD}}\left(p_{\mathrm{r}}^{*}, n^{*}\right)$ in (58) and its approximation $\tilde{\Delta}_{\mathrm{FD}}\left(p_{\mathrm{r}}^{*}, n^{*}\right)$ in (59), with the optimal result via an exhaustive search upon (58), $\bar{\Delta}_{\mathrm{FD}}\left(p_{\mathrm{r}}^{\dagger}, n^{\dagger}\right)$, in Fig. 11. These results are plotted versus the relay's maximum transmit power $p_{\mathrm{r}}^{\max }$, and the range $\left[0, p_{\mathrm{r}}^{\max }\right]$ is discretized for the search of $p_{\mathrm{r}}^{\dagger}$. As shown in this figure, all the three results perfectly agree with each other, which confirms the validity of the approximation (59) and our iterative algorithm.

In Fig. 12, we compare the minimised average AoI of HD-DF relaying, $\bar{\Delta}_{\mathrm{HD}}\left(n_{1}^{*}, n_{2}^{*}\right)$, with that of FD-DF relaying, 


$$
\begin{aligned}
& O_{4}^{\prime}(n)=\frac{3+\bar{\varepsilon}_{\mathrm{r}}^{\mathrm{nSI}}+4 n b^{\prime} / \bar{\gamma}_{\mathrm{sr}}}{2\left(1-\bar{\varepsilon}_{\mathrm{r}}^{\mathrm{nSI}}\right)}+\frac{1}{1-\bar{\varepsilon}_{\mathrm{d}}}[\underbrace{\frac{1+\bar{\varepsilon}_{\mathrm{r}}^{\mathrm{nSI}}}{1+\bar{\varepsilon}_{\mathrm{r}}^{\mathrm{SI}}}}_{\geq-1}+\frac{3 n b^{\prime}}{\bar{\gamma}_{\mathrm{d}}}+\frac{n b^{\prime}}{\bar{\gamma}_{\mathrm{d}}} \underbrace{\frac{\left(1-\bar{\varepsilon}_{\mathrm{r}}^{\mathrm{SI}}\right)}{\left(1+\bar{\varepsilon}_{\mathrm{r}}^{\mathrm{SI}}\right)}}_{<1}+\frac{n b^{\prime}\left(1+\rho b+\rho \bar{\gamma}_{\mathrm{sr}}\right)}{\bar{\gamma}_{\mathrm{sr}}(1+\rho b)} \underbrace{\frac{\left(1-\bar{\varepsilon}_{\mathrm{r}} \mathrm{SI}\right)}{\left(1+\bar{\varepsilon}_{\mathrm{r}}^{\mathrm{SI}}\right)}}_{<1} \underbrace{-\frac{n b^{\prime}\left(1-\bar{\varepsilon}_{\mathrm{r}}^{\mathrm{nSI}}\right)}{\left(1+\bar{\varepsilon}_{\mathrm{r}}^{\mathrm{SI}}\right) \bar{\gamma}_{\mathrm{sr}}}}_{>0}] \\
& +\underbrace{\frac{1-\bar{\varepsilon}_{\mathrm{r}}^{\mathrm{SI}}}{\left(1-\bar{\varepsilon}_{\mathrm{r}}^{\mathrm{nSI}}\right)\left(1-\bar{\varepsilon}_{\mathrm{d}}\right)}}_{<1 /\left(1-\bar{\varepsilon}_{\mathrm{d}}\right)}[-1+\frac{n b^{\prime}\left(1+\rho b+\rho \bar{\gamma}_{\mathrm{sr}}\right)}{\bar{\gamma}_{\mathrm{sr}}(1+\rho b)} \underbrace{\frac{n b^{\prime}}{\bar{\gamma}_{\mathrm{d}}}-\frac{n b^{\prime}}{\bar{\gamma}_{\mathrm{sr}}}}_{>0}] \underbrace{-\frac{\rho b\left(1-\bar{\varepsilon}_{\mathrm{r}}^{\mathrm{SI}}\right)^{2}}{\left(1-\bar{\varepsilon}_{\mathrm{d}}\right)\left(1+\bar{\varepsilon}_{\mathrm{r}}^{\mathrm{SI}}\right)^{2}}\left[\frac{n b^{\prime}}{\bar{\gamma}_{\mathrm{d}}}+\frac{n b^{\prime}\left(1+\rho b+\rho \bar{\gamma}_{\mathrm{sr}}\right)}{(1+\rho b) \bar{\gamma}_{\mathrm{sr}}}\right]}_{>0} \\
& \underbrace{-\frac{2 n b^{\prime}\left(1+\bar{\varepsilon}_{\mathrm{r}}^{\mathrm{nSI}}\right)}{\bar{\gamma}_{\mathrm{d}}\left(1-\bar{\varepsilon}_{\mathrm{d}}\right)\left(1+\bar{\varepsilon}_{\mathrm{r}}^{\mathrm{SI}}\right)^{2}}}_{>0}>\frac{3+4 n b^{\prime} / \bar{\gamma}_{\mathrm{sr}}}{2\left(1-\bar{\varepsilon}_{\mathrm{r}}^{\mathrm{nSI}}\right)}+\frac{1}{1-\bar{\varepsilon}_{\mathrm{d}}}\left[1+\frac{4 n b^{\prime}}{\bar{\gamma}_{\mathrm{d}}}+\frac{2 n b^{\prime}}{\bar{\gamma}_{\mathrm{sr}}}\left(1+\frac{\rho \bar{\gamma}_{\mathrm{sr}}}{1+\rho b}\right)\right] \\
& >\frac{3+4 n b^{\prime} / \bar{\gamma}_{\mathrm{sr}}}{2\left(1-\bar{\varepsilon}_{\mathrm{r}}^{\mathrm{nSI}}\right)}+\frac{1}{1-\bar{\varepsilon}_{\mathrm{d}}}\left[1+\frac{4 n b^{\prime}}{\bar{\gamma}_{\mathrm{d}}}+\frac{2 n b^{\prime}}{\bar{\gamma}_{\mathrm{sr}}}\left(1+\rho \bar{\gamma}_{\mathrm{sr}}\right)\right] \text {. }
\end{aligned}
$$

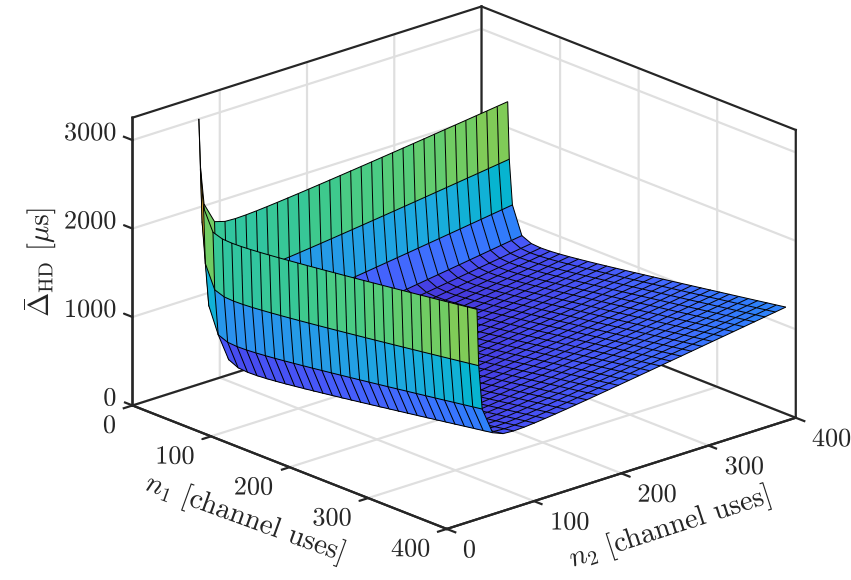

Fig. 8. The average AoI of the HD-DF relaying IoT, $\bar{\Delta}_{\mathrm{HD}}$ in (21), versus the packet blocklengths $n_{1}$ and $n_{2}$ in Phases 1 and 2, with $p_{\mathrm{s}}=5 \mathrm{dBm}$ and $p_{\mathrm{r}}=15 \mathrm{dBm}$.

$\bar{\Delta}_{\mathrm{FD}}\left(p_{\mathrm{r}}^{*}, n^{*}\right)$, which are both reduced as the IoT device's transmit power $p_{\mathrm{s}}$ increases. In the HD-DF relaying, the average packet error probability $\bar{\varepsilon}_{\mathrm{r}}$ decreases upon increasing $p_{\mathrm{s}}$ and, consequently, the number of total (re)transmissions from the IoT device to the relay is reduced, which shortens the time for the delivery of status updates. In the FD-DF relaying, the increase of $p_{\mathrm{s}}$ causes a decrease of average packet error probabilities $\bar{\varepsilon}_{\mathrm{r}}^{\mathrm{SI}}$ and $\bar{\varepsilon}_{\mathrm{r}}^{\mathrm{nSI}}$, which is ultimately reflected by the decrease of average AoI. As shown in the minimised average AoI comparisons between the HD-DF and the FD-DF relaying, the average AoI of FD-DF relaying is smaller than that of HD-DF relaying when $p_{\mathrm{s}}$ is above a threshold value, given the relay's SI cancellation capability which pertains to the value of $H_{\text {rr }}$. Moreover, the threshold for $p_{\mathrm{s}}$ is lowered upon enhancing the relay's SI cancellation capability, which provides an instructive suggestion on how to select a relaying scheme based on the IoT device's transmit power and the relay's SI cancellation capability.

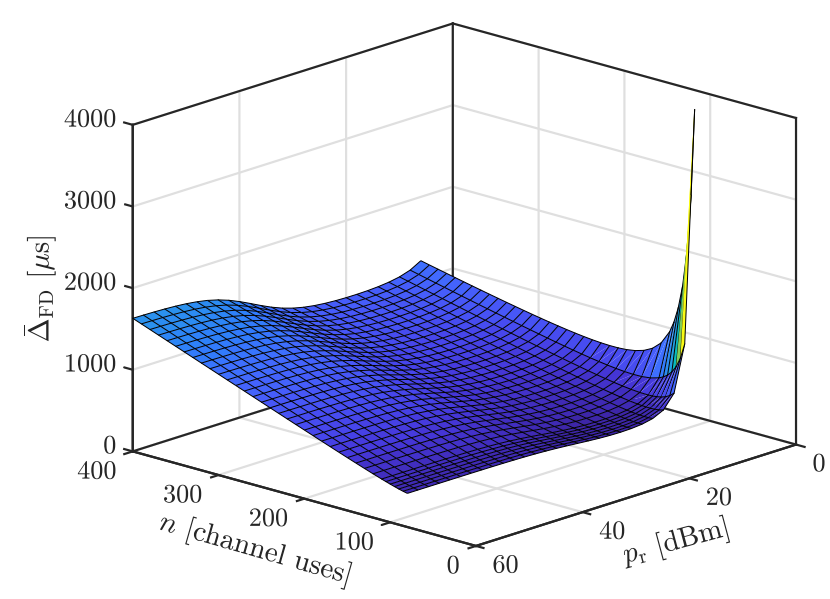

Fig. 9. The average AoI of the FD-DF relaying IoT, $\bar{\Delta}_{\mathrm{FD}}$ in (54), versus the packet blocklength $n$ and the relay's transmit power $p_{\mathrm{r}}$, with $p_{\mathrm{s}}=5 \mathrm{dBm}$ and $H_{\mathrm{rr}}=-115 \mathrm{~dB}$.

A special case in Fig. 12 is the result $\bar{\Delta}_{\mathrm{FD}}\left(p_{\mathrm{r}}^{*}, n^{*}\right)$ with $H_{\mathrm{rr}}=$ $-90 \mathrm{~dB}$, which exhibits a two-stage decrease: a first quick fall followed by a slowing down. The main reason behind this is that the relay's SI cancellation capability is not strong enough to leave the error probability $\bar{\varepsilon}_{\mathrm{r}}^{\mathrm{SI}}$ dominated by the IoT device's transmit power $p_{\mathrm{s}}$. The first quick fall is prominently contributed by the error probability $\bar{\varepsilon}_{\mathrm{r}}^{\mathrm{nSI}}$ whose slope is large in the region of low transmit power $p_{\mathrm{s}}$. The second stage is caused by the joint contribution of $\bar{\varepsilon}_{\mathrm{r}}^{\mathrm{nSI}}$ and $\bar{\varepsilon}_{\mathrm{r}}^{\mathrm{SI}}$ whose slopes both decrease as $p_{\mathrm{s}}$ increases.

In addition, the impact of the relay's maximum transmit power $p_{\mathrm{r}}^{\max }$ on the minimised average AoI in both HD-DF and FD-DF relaying IoT networks is investigated in Fig. 13, where the minimised average AoI of either HD-DF or FD-DF is decreased to a certain level as $p_{\mathrm{r}}^{\max }$ increases. As implied by its convergence property, the minimised average AoI will not be influenced by $p_{\mathrm{r}}^{\max }$ if the latter is sufficiently high. Moreover, similar with the observation in Fig. 12, the stronger 


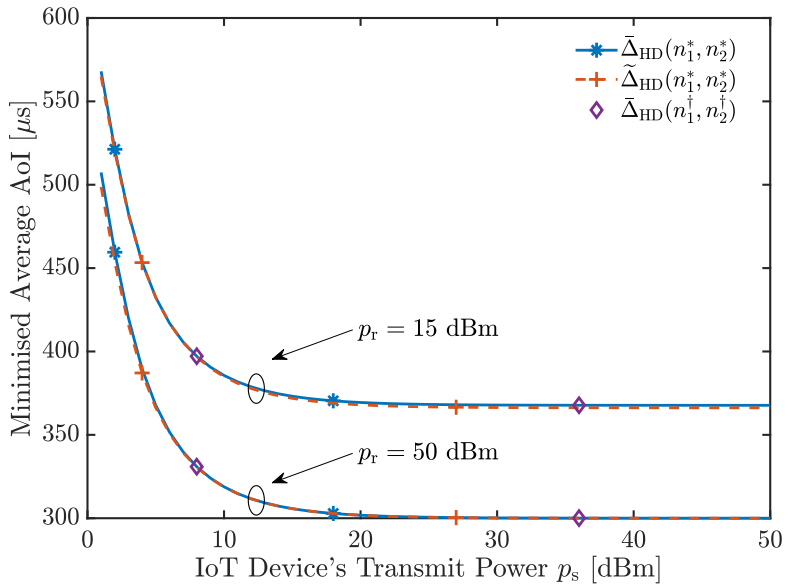

Fig. 10. Comparisons between the minimised average AoI with the solutions in (36) and (38), $\bar{\Delta}_{\mathrm{HD}}\left(n_{1}^{*}, n_{2}^{*}\right)$ given by (21) and its approximation $\tilde{\Delta}_{\mathrm{HD}}\left(n_{1}^{*}, n_{2}^{*}\right)$ given by (23). Also shown is $\bar{\Delta}_{\mathrm{HD}}\left(n_{1}^{\dagger}, n_{2}^{\dagger}\right)$ with the optimal solution via exhaustive search upon (21).

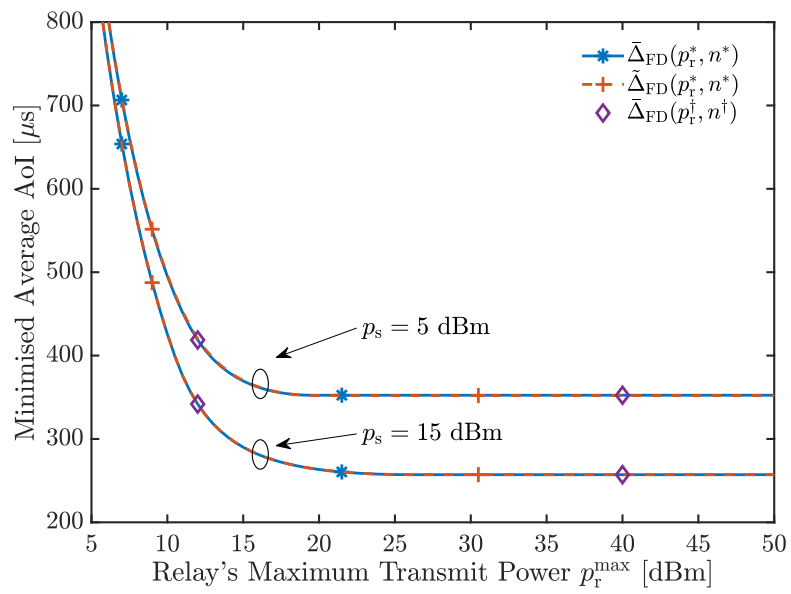

Fig. 11. Comparisons between the minimised average AoI with the solutions by our proposed iterative algorithm, $\bar{\Delta}_{\mathrm{FD}}\left(p_{\mathrm{r}}^{*}, n^{*}\right)$ given by $(58)$ and its approximation $\tilde{\Delta}_{\mathrm{FD}}\left(p_{\mathrm{r}}^{*}, n^{*}\right)$ given by (59). Also shown is $\bar{\Delta}_{\mathrm{FD}}\left(p_{\mathrm{r}}^{\dagger}, n^{\dagger}\right)$ with the optimal solution via exhaustive search upon (58).

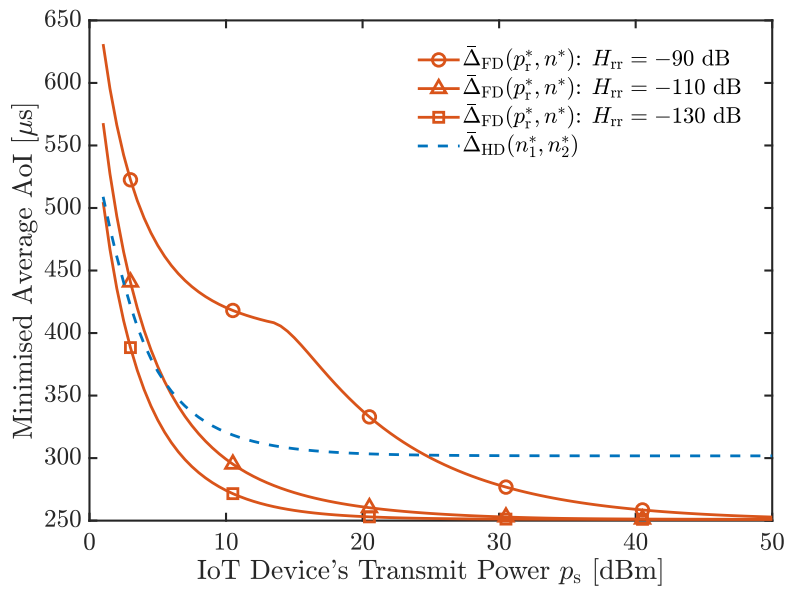

Fig. 12. Minimised average AoI comparisons between HD-DF and FD-DF relaying versus the IoT device's transmit power $p_{\mathrm{s}}$, with $p_{\mathrm{r}}^{\max }=30 \mathrm{dBm}$.

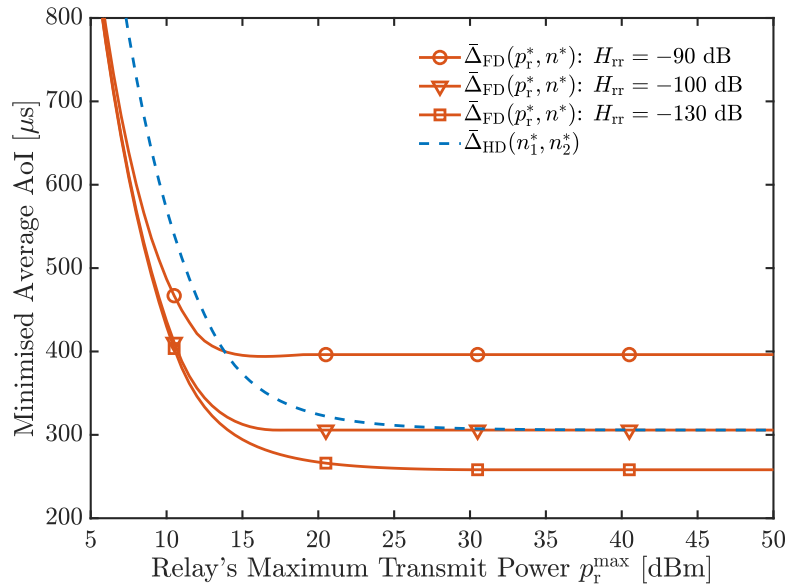

Fig. 13. Minimised average AoI comparisons between HD-DF and FD-DF relaying versus the relay's maximum transmit power $p_{\mathrm{r}}^{\max }$, with $p_{\mathrm{s}}=15$ $\mathrm{dBm}$.

is the relay's SI cancellation capability, the smaller is the minimised average AoI of the FD-DF relaying. Further, given $p_{\mathrm{r}}^{\max }$, the FD-DF relaying outperforms the HD-DF relaying when the relay's SI cancellation capability is above certain threshold. Note that, when $p_{\mathrm{r}}^{\max }$ is large enough, the relay's SI cancellation capability preserves its dominant position in the minimised average AoI of FD-DF relaying, which is owing to the damage caused by SI in the relay's decoding of its received status packets from the IoT device.

\section{CONCLUSIONS}

To achieve timely status updates in IoT monitoring networks, short-packet relaying has been investigated in this paper, where the status updates generated at an IoT device are delivered to a remote server with the aid of a relay in both HD-DF and FD-DF modes. For quantifying the data freshness of status updates, we formulated the average AoI for both relaying modes in finite blocklength regime. For the HD-DF relaying, the optimisation problem was established to minimise the average AoI with optimal blocklengths for the delivery of a status update in Phases 1 and 2, which was solved through a perfect approximation of the average AoI. For the HD-DF relaying, the optimisation problem was built on the basis of the average AoI minimisation through optimal designs of the blocklength and the relay's transmit power, which was solved by our proposed iterative algorithm. Illustrative numerical results substantiated the validity of our theoretical formulations and the feasibility of our proposed algorithms. Moreover, the minimised average AoI comparisons between HD-DF and FD-DF relaying IoT networks have shown that the latter outperforms the former if the relay has a strong SI cancellation capability. In other words, the utilisation of FD mode will help with the improvement of data freshness in the IoT networks, as long as sufficient SI has been cancelled.

\section{APPENDIX A}

PROOF OF LEMMA 2

According to the law of total probability, the probability of $K_{\mathrm{r}}=k$ is given in (80), where $K_{\mathrm{d}}$ denotes the number of total 


$$
\begin{aligned}
& \operatorname{Pr}\left(K_{\mathrm{r}}=k\right)=\sum_{k^{\prime}=1}^{k-1} \operatorname{Pr}\left(K_{\mathrm{r}}=k \mid K_{\mathrm{d}}=k^{\prime}\right) \operatorname{Pr}\left(K_{\mathrm{d}}=k^{\prime}\right)+\sum_{k^{\prime}=k}^{\infty} \operatorname{Pr}\left(K_{\mathrm{r}}=k \mid K_{\mathrm{d}}=k^{\prime}\right) \operatorname{Pr}\left(K_{\mathrm{d}}=k^{\prime}\right) \\
& =\sum_{k^{\prime}=1}^{k-1} \bar{\varepsilon}_{\mathrm{d}}^{k^{\prime}-1}\left(1-\bar{\varepsilon}_{\mathrm{d}}\right)\left(\bar{\varepsilon}_{\mathrm{r}}^{\mathrm{SI}}\right)^{k^{\prime}}\left(\bar{\varepsilon}_{\mathrm{r}}^{\mathrm{nSI}}\right)^{k-1-k^{\prime}}\left(1-\bar{\varepsilon}_{\mathrm{r}}^{\mathrm{nSI}}\right)+\sum_{k^{\prime}=k}^{\infty} \bar{\varepsilon}_{\mathrm{d}}^{k^{\prime}-1}\left(1-\bar{\varepsilon}_{\mathrm{d}}\right)\left(\bar{\varepsilon}_{\mathrm{r}}^{\mathrm{SI}}\right)^{k-1}\left(1-\bar{\varepsilon}_{\mathrm{r}}^{\mathrm{SI}}\right)
\end{aligned}
$$

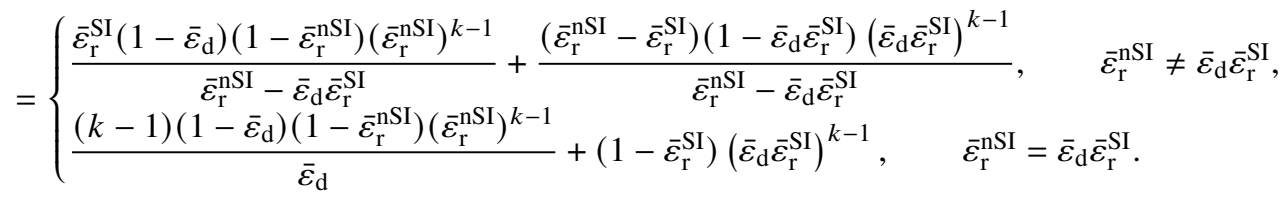

(re)transmissions from the relay to the remote server for a single status update decoded successfully at the remote server without preemption at the relay.

Thus, the mean of $K_{\mathrm{r}}$ is calculated using

$$
\begin{aligned}
& \mathbb{E}\left(K_{\mathrm{r}}\right)=\sum_{k=1}^{\infty} k \operatorname{Pr}\left(K_{\mathrm{r}}=k\right) \\
& =\frac{\bar{\varepsilon}_{\mathrm{r}}^{\mathrm{SI}}\left(1-\bar{\varepsilon}_{\mathrm{d}}\right)\left(1-\bar{\varepsilon}_{\mathrm{r}}^{\mathrm{nSI}}\right)}{\bar{\varepsilon}_{\mathrm{r}}^{\mathrm{nSI}}-\bar{\varepsilon}_{\mathrm{d}} \bar{\varepsilon}_{\mathrm{r}}^{\mathrm{SI}}} \sum_{k=1}^{\infty} k\left(\bar{\varepsilon}_{\mathrm{r}}^{\mathrm{nSI}}\right)^{k-1} \\
& +\frac{\left(\bar{\varepsilon}_{\mathrm{r}}^{\mathrm{nSI}}-\bar{\varepsilon}_{\mathrm{r}}^{\mathrm{SI}}\right)\left(1-\bar{\varepsilon}_{\mathrm{d}} \bar{\varepsilon}_{\mathrm{r}}^{\mathrm{SI}}\right)}{\bar{\varepsilon}_{\mathrm{r}}^{\mathrm{nSI}}-\bar{\varepsilon}_{\mathrm{d}} \bar{\varepsilon}_{\mathrm{r}}^{\mathrm{SI}}} \sum_{k=1}^{\infty} k\left(\bar{\varepsilon}_{\mathrm{d}} \bar{\varepsilon}_{\mathrm{r}}^{\mathrm{SI}}\right)^{k-1} \\
& =\frac{1+\bar{\varepsilon}_{\mathrm{r}}^{\mathrm{SI}}-\bar{\varepsilon}_{\mathrm{r}}^{\mathrm{nSI}}-\bar{\varepsilon}_{\mathrm{d}} \bar{\varepsilon}_{\mathrm{r}}^{\mathrm{SI}}}{\left(1-\bar{\varepsilon}_{\mathrm{r}}^{\mathrm{nSI}}\right)\left(1-\bar{\varepsilon}_{\mathrm{d}} \bar{\varepsilon}_{\mathrm{r}}^{\mathrm{SI}}\right)},
\end{aligned}
$$

where $\bar{\varepsilon}_{\mathrm{r}}^{\mathrm{nSI}} \neq \bar{\varepsilon}_{\mathrm{d}} \bar{\varepsilon}_{\mathrm{r}}^{\mathrm{SI}}$. In the case that $\bar{\varepsilon}_{\mathrm{r}}^{\mathrm{nSI}}=\bar{\varepsilon}_{\mathrm{d}} \bar{\varepsilon}_{\mathrm{r}}^{\mathrm{SI}}$, (81) is recalculated as

$$
\begin{aligned}
& \mathbb{E}\left(K_{\mathrm{r}}\right)=\frac{\left(1-\bar{\varepsilon}_{\mathrm{d}}\right)\left(1-\bar{\varepsilon}_{\mathrm{r}}^{\mathrm{nSI}}\right)}{\bar{\varepsilon}_{\mathrm{d}}} \sum_{k=1}^{\infty} k(k-1)\left(\bar{\varepsilon}_{\mathrm{r}}^{\mathrm{nSI}}\right)^{k-1} \\
& +\left(1-\bar{\varepsilon}_{\mathrm{r}}^{\mathrm{SI}}\right) \sum_{k=1}^{\infty} k\left(\bar{\varepsilon}_{\mathrm{d}} \bar{\varepsilon}_{\mathrm{r}}^{\mathrm{SI}}\right)^{k-1}=\frac{1+\bar{\varepsilon}_{\mathrm{r}}^{\mathrm{SI}}-2 \bar{\varepsilon}_{\mathrm{r}}^{\mathrm{nSI}}}{\left(1-\bar{\varepsilon}_{\mathrm{r}}^{\mathrm{nSI}}\right)^{2}} .
\end{aligned}
$$

We remark that, (81) and (82) are equivalent to the same expression. As a general result, the mean of $K_{\mathrm{r}}$ is expressed as (49) in Lemma 2.

The second moment of $K_{\mathrm{r}}$ is calculated using

$$
\begin{aligned}
\mathbb{E} & \left(K_{\mathrm{r}}^{2}\right)=\sum_{k=1}^{\infty} k^{2} \operatorname{Pr}\left(K_{\mathrm{r}}=k\right) \\
= & \frac{\bar{\varepsilon}_{\mathrm{r}}^{\mathrm{SI}}\left(1-\bar{\varepsilon}_{\mathrm{d}}\right)\left(1-\bar{\varepsilon}_{\mathrm{r}}^{\mathrm{nSI}}\right)}{\bar{\varepsilon}_{\mathrm{r}}^{\mathrm{nSI}}-\bar{\varepsilon}_{\mathrm{d}} \bar{\varepsilon}_{\mathrm{r}}^{\mathrm{SI}}} \sum_{k=1}^{\infty} k^{2}\left(\bar{\varepsilon}_{\mathrm{r}}^{\mathrm{nSI}}\right)^{k-1} \\
& +\frac{\left(\bar{\varepsilon}_{\mathrm{r}}^{\mathrm{nSI}}-\bar{\varepsilon}_{\mathrm{r}}^{\mathrm{SI}}\right)\left(1-\bar{\varepsilon}_{\mathrm{d}} \bar{\varepsilon}_{\mathrm{r}}^{\mathrm{SI}}\right)}{\bar{\varepsilon}_{\mathrm{r}}^{\mathrm{nSI}}-\bar{\varepsilon}_{\mathrm{d}} \bar{\varepsilon}_{\mathrm{r}}^{\mathrm{SI}}} \sum_{k=1}^{\infty} k^{2}\left(\bar{\varepsilon}_{\mathrm{d}} \bar{\varepsilon}_{\mathrm{r}}^{\mathrm{SI}}\right)^{k-1} \\
= & \frac{\left(1+\bar{\varepsilon}_{\mathrm{r}}^{\mathrm{SI}}-\bar{\varepsilon}_{\mathrm{r}}^{\mathrm{nSI}}-\bar{\varepsilon}_{\mathrm{d}} \bar{\varepsilon}_{\mathrm{r}}^{\mathrm{SI}}\right)}{\left(1-\bar{\varepsilon}_{\mathrm{r}}^{\mathrm{nSI}}\right)\left(1-\bar{\varepsilon}_{\mathrm{d}} \bar{\varepsilon}_{\mathrm{r}}^{\mathrm{SI}}\right)}\left(\frac{2}{1-\bar{\varepsilon}_{\mathrm{r}}^{\mathrm{nSI}}}+\frac{2}{1-\bar{\varepsilon}_{\mathrm{d}} \bar{\varepsilon}_{\mathrm{r}}^{\mathrm{SI}}}-1\right) \\
& -\frac{2}{\left(1-\bar{\varepsilon}_{\mathrm{r}}^{\mathrm{nSI}}\right)\left(1-\bar{\varepsilon}_{\mathrm{d}} \bar{\varepsilon}_{\mathrm{r}}^{\mathrm{SI}}\right)},
\end{aligned}
$$

where $\bar{\varepsilon}_{\mathrm{r}}^{\mathrm{nSI}} \neq \bar{\varepsilon}_{\mathrm{d}} \bar{\varepsilon}_{\mathrm{r}}^{\mathrm{SI}}$. In the case that $\bar{\varepsilon}_{\mathrm{r}}^{\mathrm{nSI}}=\bar{\varepsilon}_{\mathrm{d}} \bar{\varepsilon}_{\mathrm{r}}^{\mathrm{SI}},(83)$ is recalculated as

$$
\mathbb{E}\left(K_{\mathrm{r}}^{2}\right)=\frac{\left(1-\bar{\varepsilon}_{\mathrm{d}}\right)\left(1-\bar{\varepsilon}_{\mathrm{r}}^{\mathrm{nSI}}\right)}{\bar{\varepsilon}_{\mathrm{d}}} \sum_{k=1}^{\infty} k^{2}(k-1)\left(\bar{\varepsilon}_{\mathrm{r}}^{\mathrm{nSI}}\right)^{k-1}
$$

$$
\begin{aligned}
& +\left(1-\bar{\varepsilon}_{\mathrm{r}}^{\mathrm{SI}}\right) \sum_{k=1}^{\infty} k^{2}\left(\bar{\varepsilon}_{\mathrm{d}} \bar{\varepsilon}_{\mathrm{r}}^{\mathrm{SI}}\right)^{k-1} \\
= & \frac{\left(3+\bar{\varepsilon}_{\mathrm{r}}^{\mathrm{nSI}}\right)\left(1+\bar{\varepsilon}_{\mathrm{r}}^{\mathrm{SI}}-2 \bar{\varepsilon}_{\mathrm{r}}^{\mathrm{nSI}}\right)}{\left(1-\bar{\varepsilon}_{\mathrm{r}}^{\mathrm{nSI}}\right)^{3}}-\frac{2}{\left(1-\bar{\varepsilon}_{\mathrm{r}}^{\mathrm{nSI}}\right)^{2}} .
\end{aligned}
$$

As (83) and (84) are equivalent to the same expression, the second moment of $K_{\mathrm{r}}$ is expressed as (50) in Lemma 2.

\section{APPENDIX B}

\section{PROOF OF LEMMA 3}

The mean of $M$ is formulated as

$$
\begin{aligned}
& \mathbb{E}(M)=\mathbb{E}\left(X \mid K_{\mathrm{r}} \geqslant K_{\mathrm{d}}\right)+\sum_{l=0}^{\infty} \eta^{l}(1-\eta) l \mathbb{E}\left(X \mid K_{\mathrm{r}}<K_{\mathrm{d}}\right) \\
& =\mathbb{E}\left(X \mid K_{\mathrm{r}} \geqslant K_{\mathrm{d}}\right)+\frac{\eta}{1-\eta} \mathbb{E}\left(X \mid K_{\mathrm{r}}<K_{\mathrm{d}}\right) \\
& \stackrel{(a)}{=} \frac{\mathbb{E}(X)}{1-\eta}=\frac{\mathbb{E}\left(K_{\mathrm{r}}\right) T_{\mathrm{FD}}}{1-\eta},
\end{aligned}
$$

where $(a)$ holds according to the law of total expectation, and $\eta$ denotes the probability that the relay's currently decoded status update is preempted by the succeeding one.

The second moment of $M$ is calculated using

$$
\begin{aligned}
\mathbb{E}\left(M^{2}\right)= & \mathbb{E}\left(X^{2} \mid K_{\mathrm{r}} \geqslant K_{\mathrm{d}}\right)+\sum_{l=0}^{\infty} \eta^{l}(1-\eta) l \mathbb{E}\left(X^{2} \mid K_{\mathrm{r}}<K_{\mathrm{d}}\right) \\
& +\sum_{l=0}^{\infty} \eta^{l}(1-\eta) 2 l \mathbb{E}\left(X \mid K_{\mathrm{r}} \geqslant K_{\mathrm{d}}\right) \mathbb{E}\left(X \mid K_{\mathrm{r}}<K_{\mathrm{d}}\right) \\
& +\sum_{l=0}^{\infty} \eta^{l}(1-\eta) l(l-1) \mathbb{E}^{2}\left(X \mid K_{\mathrm{r}}<K_{\mathrm{d}}\right) \\
= & \mathbb{E}\left(X^{2} \mid K_{\mathrm{r}} \geqslant K_{\mathrm{d}}\right)+\frac{\eta}{1-\eta} \mathbb{E}\left(X^{2} \mid K_{\mathrm{r}}<K_{\mathrm{d}}\right) \\
& +\frac{2 \eta}{1-\eta} \mathbb{E}\left(X \mid K_{\mathrm{r}} \geqslant K_{\mathrm{d}}\right) \mathbb{E}\left(X \mid K_{\mathrm{r}}<K_{\mathrm{d}}\right) \\
& +\frac{2 \eta^{2}}{(1-\eta)^{2}} \mathbb{E}^{2}\left(X \mid K_{\mathrm{r}}<K_{\mathrm{d}}\right) \\
= & \frac{\mathbb{E}\left(X^{2}\right)}{1-\eta}+\frac{2 \eta \mathbb{E}(X) \mathbb{E}\left(X \mid K_{\mathrm{r}}<K_{\mathrm{d}}\right)}{(1-\eta)^{2}} \\
= & {\left[\frac{\mathbb{E}\left(K_{\mathrm{r}}^{2}\right)}{1-\eta}+\frac{2 \eta \mathbb{E}\left(K_{\mathrm{r}}\right) \mathbb{E}\left(K_{\mathrm{r}} \mid K_{\mathrm{r}}<K_{\mathrm{d}}\right)}{(1-\eta)^{2}}\right] T_{\mathrm{FD}}^{2} . }
\end{aligned}
$$

\section{REFERENCES}

[1] A. Al-Fuqaha, M. Guizani, M. Mohammadi, M. Aledhari, and M. Ayyash, "Internet of things: A survey on enabling technologies, pro- 
tocols, and applications," IEEE Commun. Surveys Tuts., vol. 17, no. 4, pp. 2347-2376, 4th Quart., 2015.

[2] E. Ahmed, I. Yaqoob, A. Gani, M. Imran, and M. Guizani, "Internet-ofthings-based smart environments: State of the art, taxonomy, and open research challenges," IEEE Wireless Commun., vol. 23, no. 5, pp. 10-16, Oct. 2016.

[3] F. Montori, L. Bedogni, and L. Bononi, "A collaborative internet of things architecture for smart cities and environmental monitoring," IEEE Internet Things J., vol. 5, no. 2, pp. 592-605, Apr. 2018.

[4] R. Du, P. Santi, M. Xiao, A. V. Vasilakos, and C. Fischione, "The sensable city: A survey on the deployment and management for smart city monitoring," IEEE Commun. Surveys Tuts., vol. 21, no. 2, pp. 15331560, 2nd Quart. 2019.

[5] Y. Gu, H. Chen, Y. Zhou, Y. Li, and B. Vucetic, "Timely status update in internet of things monitoring systems: An age-energy tradeoff," IEEE Internet Things J., vol. 6, no. 3, pp. 5324-5335, Jun. 2019.

[6] M. A. Abd-Elmagid, N. Pappas, and H. S. Dhillon, "On the role of age of information in the internet of things," IEEE Commun. Mag., vol. 57, no. 12, pp. 72-77, Dec. 2019.

[7] S. Kaul, M. Gruteser, V. Rai, and J. Kenney, "Minimizing age of information in vehicular networks," in Proc. IEEE Conf. Sensor, Mesh Ad Hoc Commun. Netw. (SECON), Salt Lake City, UT, USA, Jun. 2011, pp. $350-358$.

[8] S. Kaul, R. Yates, and M. Gruteser, "Real-time status: How often should one update?," in Proc. IEEE INFOCOM, Orlando, FL, USA, Mar. 2012, pp. $2731-2735$.

[9] A. Kosta, N. Pappas, and V. Angelakis, "Age of information: A new concept, metric, and tool," Found. Trends Netw., vol. 12, no. 3, pp. 162-259, 2017.

[10] R. D. Yates, Y. Sun, D. Richard Brown, S. K. Kaul, E. Modiano and S. Ulukus, "Age of information: An introduction and survey," IEEE J. Sel. Areas Commun., vol. 39, no. 5, pp. 1183-1210, May 2021.

[11] A. Soysal and S. Ulukus, "Age of information in G/G/1/1 systems: Age expressions, bounds, special cases, and optimization," May 2019, arXiv:1905.13743. [Online]. Available: https://arxiv.org/abs/1905.13743.

[12] R. D. Yates, "The age of gossip in networks," Feb. 2020 arXiv:2012.02893. [Online]. Available: https://arxiv.org/abs/2102.02893.

[13] P. Rafiee, P. Zou, O. Ozel and S. Subramaniam, "Maintaining information freshness in power-efficient status update systems," in Proc. IEEE Conf. Comput. Commun. Workshops (INFOCOM WKSHPS), Toronto, ON, Canada, Jul. 2020, pp. 31-36.

[14] I. Krikidis, "Average age of information in wireless powered sensor networks," IEEE Wireless Commun. Lett., vol. 8, no. 2, pp. 628-631, Apr. 2019.

[15] A. Arafa and S. Ulukus, "Timely updates in energy harvesting two-hop networks: Offline and online policies," IEEE Trans. Wireless Commun., vol. 18, no. 8, pp. 4017-4030, Aug. 2019.

[16] O. Ozel, "Timely status updating through intermittent sensing and transmission," in Proc. IEEE Int. Symp. Inf. Theory (ISIT), Los Angeles, CA, USA, Jun. 2020, pp. 1788-1793.

[17] R. V. Bhat, R. Vaze, and M. Motani, "Throughput maximization with an average age of information constraint in fading channels," IEEE Trans. Wireless Commun., vol. 20, no. 1, pp. 481-494, Jan. 2021.

[18] A. Arafa, K. Banawan, K. G. Seddik, and H. Vincent Poor, "Timely estimation using coded quantized samples," in Proc. IEEE Int. Symp. Inf. Theory (ISIT), Los Angeles, CA, USA, Jun. 2020, pp. 1812-1817.

[19] P. Mayekar, P. Parag, and H. Tyagi, "Optimal source codes for timely updates," IEEE Trans. Inf. Theory, vol. 66, no. 6, pp. 3714-3731, Jun. 2020.

[20] A. Arafa, K. Banawan, K. G. Seddik, and H. V. Poor, "On timely channel coding with hybrid ARQ," in Proc. IEEE Global Commun. Conf. (GLOBECOM), Waikoloa, HI, USA, Dec. 2019, pp. 1-6.

[21] M. Bastopcu and S. Ulukus, "Partial updates: Losing information for freshness," in Proc. IEEE Int. Symp. Inf. Theory (ISIT), Los Angeles, CA, USA, Jun. 2020, pp. 1800-1805.

[22] M. Bastopcu, B. Buyukates, and S. Ulukus, "Selective encoding policies for maximizing information freshness," IEEE Transactions on Communications, early access, doi: 10.1109/TCOMM.2021.3059871.

[23] A. Maatouk, M. Assaad, and A. Ephremides, "The age of incorrect information: An enabler of semantics-empowered communication," Dec. 2020, arXiv:2012.13214. [Online]. Available: https://arxiv.org/abs/2012.13214.

[24] Y. Yang, "Permutation-based transmissions in ultra-reliable and lowlatency communications," IEEE Commun. Lett., vol. 25, no. 3, pp. 1024 1028, Mar. 2021.
[25] Y. Yang and L. Hanzo, "Permutation-based TCP and UDP transmissions to improve goodput and latency in the internet-of-things," IEEE Internet Things J., doi: 10.1109/JIOT.2021.3068238.

[26] G. Durisi, T. Koch, and P. Popovski, "Toward massive, ultrareliable, and low-latency wireless communication with short packets," Proc. IEEE, vol. 104, no. 9, pp. 1711-1726, Sept. 2016.

[27] Y. Polyanskiy, H. V. Poor, and S. Verdu, "Channel coding rate in the finite blocklength regime," IEEE Trans. Inf. Theory, vol. 56, no. 5, pp. 2307-2359, May 2010.

[28] R. Wang, Y. Gu, H. Chen, Y. Li, and B. Vucetic, "On the age of information of short-packet communications with packet management," in Proc. IEEE Global Commun. Conf. (GLOBECOM), Waikoloa, HI, USA, 2019, pp. 1-6.

[29] H. Pan and S. C. Liew, "Information update: TDMA or FDMA?," IEEE Wireless Commun. Lett., vol. 9, no. 6, pp. 856-860, Jun. 2020.

[30] B. Yu, Y. Cai, D. Wu, and Z. Xiang, "Average age of information in short packet based machine type communication," IEEE Trans. Veh. Technol., vol. 69, no. 9, pp. 10306-10319, Sept. 2020.

[31] M. Kamel, W. Hamouda, and A. Youssef, "Ultra-dense networks: A survey," IEEE Commun. Surveys Tuts., vol. 18, no. 4, pp. 2522-2545, 4th Quart., 2016.

[32] X. Liu and N. Ansari, "Green relay assisted D2D communications with dual batteries in heterogeneous cellular networks for IoT," IEEE Internet Things J., vol. 4, no. 5, pp. 1707-1715, Oct. 2017.

[33] H. Zhang, H. Liu, J. Cheng, and V. C. M. Leung, "Downlink energy efficiency of power allocation and wireless backhaul bandwidth allocation in heterogeneous small cell networks," IEEE Trans. Commun., vol. 66, no. 4, pp. 1705-1716, Apr. 2018.

[34] B. Buyukates, A. Soysal, and S. Ulukus, "Age of information in multihop multicast networks," J. Commun. Netw, vol. 21, no. 3, pp. 256-267, Jun. 2019.

[35] B. Li, H. Chen, N. Pappas, and Y. Li, "Optimizing information freshness in two-way relay networks," in Proc. IEEE/CIC Int. Conf. Commun. China (ICCC, Chongqing, China, Aug. 2020, pp. 893-898.

[36] Y. Yang and S. Aissa, "Information-guided transmission in decode-andforward relaying systems: Spatial exploitation and throughput enhancement," IEEE Trans. Wireless Commun., vol. 10, no. 7, pp. 2341-2351, Jul. 2011.

[37] Y. Yang, H. Ma, and S. Aissa, "Partial QoS-aware opportunistic relay selection over two-hop channels: End-to-end performance under spectrumsharing requirements," IEEE Trans. Veh. Techno., vol. 63, no. 8, pp. 3829-3840, Oct. 2014.

[38] Y. Yang, "Spatial modulation exploited in non-reciprocal two-way relay channels: Efficient protocols and capacity analysis," IEEE Trans. Commun., vol. 64, no. 7, pp. 2821-2834, Jul. 2016.

[39] B. Makki, T. Svensson, and M. Zorzi, "Finite block-length analysis of the incremental redundancy HARQ," IEEE Wireless Commun. Lett., vol. 3, no. 5, pp. 529-532, Oct. 2014.

[40] Y. Gu, H. Chen, Y. Li, and B. Vucetic, "Ultra-reliable short-packet communications: Half-duplex or full-duplex relaying?," IEEE Wireless Commun. Lett., vol. 7, no. 3, pp. 348-351, Jun. 2018.

[41] F. Yang, L. Shu, Y. Yang, Y. Liu and T. Gordon, "Improved coverage and connectivity via weighted node deployment in solar insecticidal lamp Internet of Things," IEEE Internet Things J., vol. 8, no. 12, pp. 1017010186, Jun. 2021.

[42] A. Goldsmith, Wireless Communications. Cambridge, U.K.: Cambridge Univ. Press, 2005.

[43] Z. Zhang, X. Chai, K. Long, A. V. Vasilakos, and L. Hanzo, "Full duplex techniques for $5 \mathrm{G}$ networks: self-interference cancellation, protocol design, and relay selection," IEEE Commun. Mag., vol. 53, no. 5, pp. 128-137, May 2015.

[44] S. Tian, M. Ma, Y. Yang, and B. Jiao, "Blind analog interference cancellation," IEEE Commun. Lett., vol. 21, no. 8, pp. 1867-1870, Aug. 2017.

[45] M. Ma, et. al., "A prototype of co-frequency co-time full duplex networking," IEEE Wireless Commun., vol. 27, no. 1, pp. 132-139, Feb. 2020.

[46] T. Riihonen, S. Werner, and R. Wichman, "Hybrid full-duplex/halfduplex relaying with transmit power adaptation," IEEE Trans. Wireless Commun., vol. 10, no. 9, pp. 3074-3085, Sep. 2011.

[47] H. Cui, M. Ma, L. Song, and B. Jiao, "Relay selection for two-way full duplex relay networks with amplify-and-forward protocol," IEEE Trans. Wireless Commun., vol. 13, no. 7, pp. 3768-3777, Jul. 2014.

[48] T. Kwon, S. Lim, S. Choi, and D. Hong, "Optimal duplex mode for DF relay in terms of the outage probability," IEEE Trans. Veh. Technol., vol. 59, no. 7, pp. 3628-3634, Sept. 2010. 


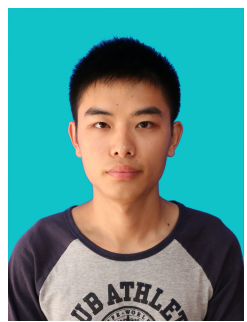

Dongsheng Zheng received the B.E. degree in microelectronic science and engineering from the University of Electronic Science and Technology of China, Chengdu, China, in 2018. He is currently pursuing the Ph.D. degree in signal and information processing with the Department of Electronics, Peking University, Beijing, China. His research interests include ultra-reliable low-latency communications, signal processing in wireless communication and information theory.

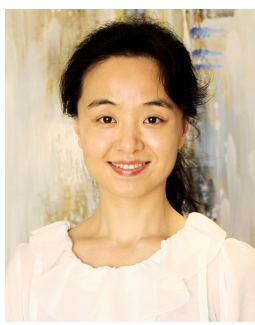

Yuli Yang (S'04-M'08-SM'19) received her Ph.D. degree in Communications \& Information Systems from Peking University in July 2007. Since Dec 2019, she has been with the University of Lincoln as a Senior Lecturer in Electrical/Electronic Engineering. From Jan 2010 to Dec 2019, she was with King Abdullah University of Science \& Technology, Melikşah University, and the University of Chester on various academic positions. Her industry experience includes working as a Research Scientist with Bell Labs Shanghai, from Aug 2007 to Dec 2009, and an Intern Researcher with Huawei Technologies, from June 2006 to July 2007. Her research interests include modelling, design, analysis and optimization of wireless systems and networks.

Lai Wei received the B.S. degree in Electronic Engineering from Peking University in 2018. He's currently pursuing the Ph.D. degree in signal and information processing with the Department of Electronics, Peking University, Beijing, China. His research interest includes wireless communication, signal processing and information theory.

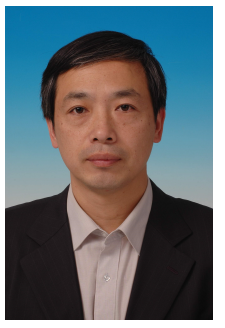

Bingli Jiao (M'05-SM'11) received the B.S. and M.S. degrees from Peking University, China, in 1983 and 1988, respectively, and the Ph.D. degree from Saarland University, Germany, in 1995 . He became an Associate Professor in 1995 and a Professor with Peking University in 2000. He currently is the director of Wireless Communication and Signal Processing Research Center, Peking University, Beijing, China. He is also a director of the Joint Laboratory for Advanced Communication Research between Peking University and Princeton University. His current research interests include full-duplex communications, information theory, and signal processing. He is a pioneer of co-frequency and co-time full-duplex as found in his early patent in 2006 . 